Westinghouse Astronuclear Laboratory

\title{
SNPOC
}

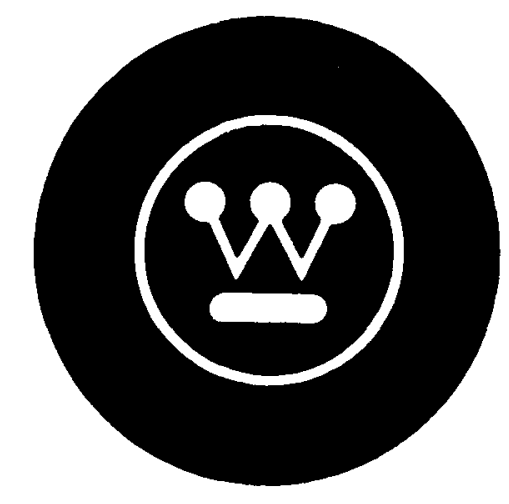

W ANL-TME- 2793 JUNE 1971

\section{MODIFICATIONS OF}

CASCADE-CASING AND RESULTS

OF CALCULATIONS 


\section{DISCLAIMER}

This report was prepared as an account of work sponsored by an agency of the United States Government. Neither the United States Government nor any agency Thereof, nor any of their employees, makes any warranty, express or implied, or assumes any legal liability or responsibility for the accuracy, completeness, or usefulness of any information, apparatus, product, or process disclosed, or represents that its use would not infringe privately owned rights. Reference herein to any specific commercial product, process, or service by trade name, trademark, manufacturer, or otherwise does not necessarily constitute or imply its endorsement, recommendation, or favoring by the United States Government or any agency thereof. The views and opinions of authors expressed herein do not necessarily state or reflect those of the United States Government or any agency thereof. 


\section{DISCLAIMER}

Portions of this document may be illegible in electronic image products. Images are produced from the best available original document. 


\section{Westinghouse Astronuclear Laboratory}

SUBCONTRACT NP-1

CODE IDENT NO. 14683

This report was prepared as an account of work
sponsored by the United States Government. Neither
the United States nor the United States Atomic Energy
Commission. nor any of their employees, nor any of
their contractors, subcontractors, or their employees,
makes any warranty, express or implied, or assumes any
legal liability or responsibility for the accuracy, com-
pleteness or usefulness of any information, apparatus,
product or process disclosed, or represents that its use
would not infringe privately owned rights.

\section{MODIFICATIONS OF}

\section{CASCADE-CASING AND RESULTS}

\section{OF CALCULATIONS}

\section{APPROVED BY:}

Lnw A canotan D. W. Drawbaugh, Maxager Reactor Physics \& Mathematics
WANL-TME-2793

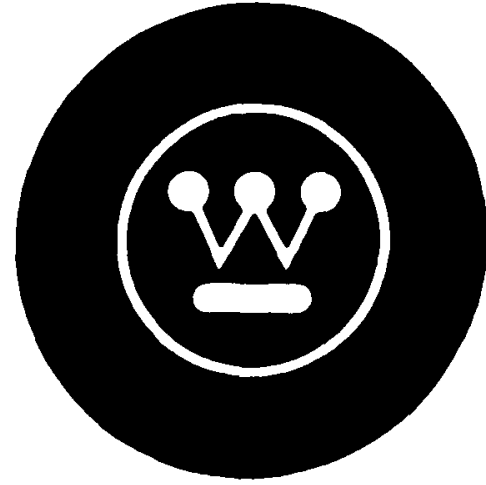

\section{JUNE 1971}




\section{PAGE BLANK}

ii 
TABLE OF CONTENTS

Section

$\underline{\text { Page }}$

1.0

INTRODUCTION

1-1

1.I REDUCTION OF COMPUTATIONAL TIME

$1-1$

1.2 OPTION FOR INPUTTING THE EXCITATION FUNCTIONS OF

$1-1$ THE DISCRETE LEVELS WHEN TREATING INELASTIC SCATTERING

1.3 NUCLEAR-LEVEL DENSITY OPTION

$1-4$

1.4 PRIMARY TRANSITION PROBABILITY OPTIONS 1-8

2.0 GAMMA RAY PRODUCTION CROSS SECTIONS FROM CASCADE-CASING;

$2-1$

INELASTIC SCATTERING OF NEUTRONS FROM VARIOUS ELEMENTS

$2.1 \quad 27 \mathrm{Al}$

$2-1$

$2.256_{\mathrm{Fe}}$

$2-3$

$2.3 \quad 48 \mathrm{TI}$

$2-4$

2.4 INELASTIC NEUTRON SCATTERING IN $\mathrm{Pb} \quad 2-4$

$2.4 .1 \quad 208_{\mathrm{Pb}} \quad 2-4$

2.4.2 $207 \mathrm{~Pb} \quad 2-6$

$2.4 .3206 \mathrm{~Pb}-$ Inelastic 2-10

3.0 GAMMA-RAY PRODUCTION MATRICES FROM CASCADE-CASING; 3-1 RADIATIVE CAPTURE OF NEUTRONS BY VARIOUS ISOTOPES

$3.148 \mathrm{Ti}(\mathrm{n}, \gamma){ }^{49} \mathrm{Ti}$

$3.2 \quad{ }^{206} \mathrm{~Pb}(\mathrm{n}, \gamma){ }^{207} \mathrm{~Pb} \quad 3-1$

$3.3207 \mathrm{~Pb}$ - n Capture 3-3

4.0 COMMENTS ON THE RESULTS 4-1

5.0 FIGURES

6.0 TABLES $6-1$ 
TABLE OF CONTENTS (continued)

Section

$\underline{\text { Page }}$

APPENDIXES

I Input Instructions for Version II of the CASCADE Code

$I-1$

II Instructions for the Use of TRANSIT

II-I

7.0

REFERENCES

$7-1$ 
W Astronuclear

Laboratory

\begin{abstract}
A number of new options have been added, and improvements made to the original $^{(1)}$ CASCADE-CASING codes. These changes are described in this report. Modified Input instructions and results of calculations for some elements of interest to the NERVA project are also presented.
\end{abstract}


(W. Astronuclear

\subsection{INTRODUCTION}

\subsection{REDUCTION OF COMPUTATIONAL TIME}

The first change was made to conserve computational time. Previously, the first $\frac{n(n+1)}{2}$ values of gamma-ray energies, $E_{\gamma}$, for which the spectrum $P\left(E_{\gamma}\right)$ was calculated corresponded to the differences in energies of the $n+l$ discrete levels. Furthermore, for plotting purposes, the next $2 \times \frac{n(n+l)}{2}$ values of $E_{\gamma}$ for which $P\left(E_{\gamma}\right)$ were calculated were obtained from $E_{\gamma}=E_{i}-E_{j} \pm \delta E$ where $i>f$ and $\delta \mathrm{E}$ is a small increment in $E_{\gamma}$. However, in manj instances, the transition probabilities, $S^{j i}$, between levels are zero. The program has been nodified so that if $S^{j i}=0, P\left(E_{\gamma}\right)$ is not evaluated for $E_{\gamma}=E_{i}-E_{j}$ nor for the two neighboring values of $\mathrm{E}_{\boldsymbol{\gamma}}$.

1.2 OPTION FOR INPUTTING THE EXCITATION FUNCTIONS OF THE DISCRETE LEVELS WHEN TREATING INELASTIC SCATTERING

In the original CASCADE code, there is an option (referred to as $S-I_{0}$ in ref. 1) which allows the partial inelastic cross sections for excitation of the discrete levels to be input provided the maximum excitation, $\epsilon$, is not in the continuum, 1.e., provided $\epsilon<\mathrm{E}_{\mathrm{m}}$. An option, which will be named S-I-2, is now available that uses these cross sections for determining the initial excitation, $r_{i}^{0}$, of the discrete levels for $\in>E_{\text {m }}$ as well as for $\in<E_{\text {m. }}$. The calculation done in the original CASCADE code (option S-I-I) can still be carried out if the cross sections for the continuum range are unknown. If option S-I-2 is used, not only the algebraic set of linear equations for finding the probability of exciting a given discrete level during a cascade is changed, but also one of the initial conditions for the fourth-order differential oquation for finding the probability of exciting lerels in the continuum is changed. 
In the following discussion, reference will be made to equations giren In WNL-ME-2744(1). Assume that a noutron-nucleus coll1sion has occurred that leaves the nuclous in an excited state, and furthermore, that the neutron has sufficiont kinotic onergy to excite the nucleus to the continum. Rather than using Eq. 40 for the initial probability, $r_{1}^{0}$, of exciting the $1^{\text {th }}$ discrete lovel option S-I-2 uses

$$
\begin{aligned}
& r_{i}^{0}=\frac{\sigma^{i}(\epsilon)}{\sigma^{\Sigma}(\epsilon)} \quad \text { for } i=1,2,3 \ldots . n \\
& r_{0}^{0}=0
\end{aligned}
$$

where $\sigma^{i}(\epsilon)$ is the cross section for exciting the $i^{\text {th }}$ level, and $\sigma^{\xi}(\epsilon)$ is the sum-total cross section for exciting all levels. Of course, if $\gamma \neq 1, \sigma^{1}$ and $\sigma^{\Sigma}$ include the cross sections for reactions such as $(n, 2 n),(n, n p)$, etc. as well as the $\left(n, n^{\prime} \gamma\right)$ reaction. The quantities $\sigma^{i}(\epsilon)$ and $\sigma^{\Sigma}(\epsilon)$ are input quantities. The quantity $\sigma^{\varepsilon}(\epsilon)$ need only be entered for $E_{m} \leq \in \leq E_{a}$ since option S-I-2 is not used for $E<\mathrm{L}_{\mathrm{m}}$ (option S-I0-1 or S-I0-2 is used in this case, depending on the value of the input-indicator IOPTN), and option S-I-I is used for $\epsilon>\mathrm{E}_{\mathrm{a}}$. The quantity $\mathrm{E}_{\mathrm{a}}$ is an input indicator. When doing a multigroup calculation, CASCADE uses option S-I-1 or S-I-2 for a particular noutron group when $\epsilon>E_{m}$ depending on whother $\epsilon\left\langle E_{a}\right.$ or $\epsilon>E_{a}$ respectively. For each nuclear level, 1 , a set of values of $\sigma^{i}(\epsilon)$ are input when required; a oufficient number of values are in the set to woll define the function $\sigma^{i}(\epsilon)$ for the energs range from threshold to a maximum excitation energy $E_{m}$ or $E_{a}$ depending on whether $\epsilon\left\langle\mathrm{F}_{\text {m }}\right.$ or $\epsilon>\mathrm{B}_{\text {m }}$ respectively. Since cross sections are normaly given as a function of the neutron energs $E_{n}=\frac{A+1}{A} \epsilon$, thes are entered into CASCADE as a function of $E_{n}$. Howerer, the calculation of a $\gamma$-rav spectrum 
in CASCADE is characterized by the maxtmun excitation onerg $\epsilon$, so the program takes into account the factor relating these onergies in interpolating to find $\sigma^{i}(\epsilon)$. Parabolic interpolation is used to determine the cross section values. For neutron energies less than the threshold onergy, i.e. $\epsilon<E_{i}, \sigma^{i}(\epsilon)=0$; also $\sigma^{\Sigma}(\epsilon)=0$ for $\epsilon<E_{1}$.

The inftial excitation probabilities are used in the ISUDS program for finding the solution to the set of linear equations. Also, when using the new option (S-I-2), the probability density $Q^{0}(E)$ for the initial excitation of energy levels in the continuum is taken to be of the same form as usod previously, soe Eq. 39.

$\frac{Q^{0}(E)}{\rho(E)}=\left(1-\frac{1}{\sigma^{E}(\epsilon)} \sum_{i=1}^{n} \sigma^{1}(\epsilon)\right)(\epsilon-E) \sigma^{c}(\epsilon-E)\left(\int_{\mathbb{E}_{\mathbf{n}}}^{\epsilon}\left(\epsilon-E^{\prime}\right) \rho\left(E^{\prime}\right) \sigma^{c}\left(\epsilon-E^{\prime}\right) d E^{\prime}\right)^{-1}$ If $\sigma^{c}(\epsilon-E)$ is treated as a constant,

$\frac{Q^{0}(E)}{\rho(E)}=\left(1-\frac{1}{\sigma^{\Sigma}(\epsilon)} \sum_{i=1}^{n} \sigma^{i}(\epsilon)\right)(\epsilon-E)\left(\int_{E_{m}}^{\epsilon}\left(\epsilon-E^{\prime}\right) \rho\left(E^{\prime}\right) d E^{\prime}\right)^{-1}$

As before, let $X(E)=\frac{Q(E)}{\rho(E)}=\psi(E)+\frac{R^{0}(E)}{\rho(E)}$

$$
\text { or } \chi=\psi+(\epsilon-E)\left(1-\frac{1}{\sigma^{\Sigma}(\epsilon)} \sum_{1=1}^{n} \sigma^{1}(\epsilon)\right)\left(\int_{E_{m}}^{\epsilon}(\epsilon-\xi) \rho(\xi) d \xi\right)^{-1}
$$

Taking derivatives with respect to $E$ :

$$
\begin{aligned}
& \chi^{\prime}=\psi^{\prime}-\left(1-\frac{1}{\sigma^{\varepsilon}(\epsilon)} \sum_{i=1}^{n} \sigma^{1}(\epsilon)\right)\left(\int_{E_{m}}^{\epsilon}(\epsilon-\xi) \rho(\xi) d \xi\right)^{-1} \\
& \chi^{\prime \prime}=\psi^{\prime \prime}, \quad \chi^{\prime \prime \prime}=\psi^{\prime \prime}, \text { and } \chi^{\prime \prime \prime \prime}=\psi^{\prime \prime \prime \prime} .
\end{aligned}
$$


Using Eq. 38, $\chi^{\prime \prime \prime \prime}=\psi^{\prime \prime \prime \prime}=6 \psi \rho_{p}+6 Q^{0_{p}}$

$$
\begin{aligned}
& \text { or } X^{\prime \prime \prime \prime}=6\left(x-\frac{R^{0}}{\rho}\right) \rho p+6 Q^{0} s=6 x \rho p \text {. } \\
& \chi(\epsilon)=0+0 \\
& x^{\prime}(\epsilon)=0-\left(1-\frac{1}{\sigma^{\Sigma}(\epsilon)}\right) \sum_{i=1}^{n} \sigma^{i}(\epsilon)\left(\int_{E_{m}}^{\epsilon}(\epsilon-\xi) \rho(\xi) d \xi\right)^{-1} \\
& x^{\prime \prime}(\epsilon)=0 \\
& \chi^{\prime \prime \prime}(\epsilon)=0
\end{aligned}
$$

The quantity $f(E)$ does not change. The above equations (for option S-I-2) are to be compared with Eggs. 53-56 (for option S-I-1). It can be seen that the second boundary condition is different.

1.3 NUCLEAR-LEVEL DENSITY OPTION

An option has been added to CASCADE to permit the use of the Gilbert and Cameron ${ }^{(2)}$ composite nuclear-level density formula (with pair and enol corrections). If one includes the entreEs dependence of the spin-dependence parameter, the density function is given by (density indicator IROE $=3$ ):

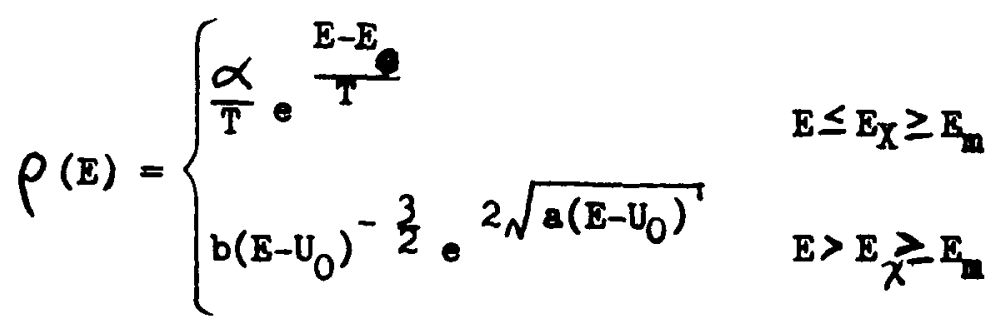

where $\alpha, a, b, E_{0}, U_{0}, T$, and $E_{X}$ are input quantities. If $b$ is sot equal to zero, the program will calculate b from

$$
b=0.198 \times a^{-\frac{1}{2}} A^{-\frac{1}{3}}
$$

$1-4$ 
where the atonde woight $A$ is an input quantity. Normally $\alpha=1$; the other paraneters can be obtained from rof. 2. The density appears in the fourthorder differential equation, one of the boundary conditions, and the normalization factor $f(E)$; see Eqs. 53-56.

The integral that appears in the boundary condition becomes: for $E_{x} \geq \epsilon \geq E_{m}$ :

$\int_{E_{m}}^{\epsilon}(\epsilon-\xi) \rho(\xi) d \xi=\frac{\alpha}{T} \int_{E_{m}}^{\epsilon}(\epsilon-E) e^{\frac{E-E_{0}}{T}} d E=\alpha\left[\left(E_{m}-\epsilon-T\right) e^{\frac{E_{m}-E_{0}}{T}}+T e^{\frac{\epsilon-E_{0}}{T}}\right] ;$ for $E_{m}<E_{x}<\epsilon:$

$$
\begin{aligned}
& \int_{E_{m}}^{\epsilon}(\epsilon-E) \rho(E) d E=\frac{\alpha}{T} \int_{E_{m}}^{E}(\epsilon-E) e^{\frac{E-E_{0}}{T}} d E+b \int_{E_{x}}^{\epsilon} \frac{(\epsilon-E)}{\left(E-U_{0}\right)^{3 / 2}} e^{2 \sqrt{a\left(E-U_{0}\right)}} d E \\
& =\alpha\left[\left(E_{m}-\epsilon-T\right) e^{\frac{E_{m}-E_{0}}{T}}+\left(\epsilon-E_{x}+T\right) e^{\frac{E_{X}-E_{0}}{T}}\right] \\
& +b\left\{\left(\frac{2\left(\epsilon-U_{0}\right)}{\sqrt{E_{X}-U_{0}}}+\frac{1}{\sqrt{a}}\right) e^{2 \sqrt{a\left(E_{X}-U_{0}\right)}}-\left(2 \sqrt{\epsilon-U_{0}}+\frac{1}{\sqrt{a}}\right) e^{2 a\left(-U_{0}\right)}+4 \sqrt{a}\left(\epsilon-U_{0}\right)[\ln x\right. \\
& \left.\left.+2 \sqrt{a} x+\frac{(2 \sqrt{a} x)^{2}}{2.2 !}+\frac{(2 \sqrt{a} x)^{3}}{3.3 !}+\ldots \frac{(2 \sqrt{a} x)^{n}}{n \cdot n !}+\frac{(2 \sqrt{a} x)^{n}}{n \cdot n !} \frac{(2 \sqrt{a} x)}{\frac{(n+1)^{2}}{n}}+\cdots\right]_{x=\sqrt{E_{X}-U_{0}}}^{x=\sqrt{\epsilon-U_{0}}}\right\}
\end{aligned}
$$

The integral that appears in the normalization factor becomes:

for $E_{X} \geq E \geq E_{m}$ :

$$
\begin{aligned}
& \int_{E_{m}}^{E}(E-\xi)^{3} p(\xi) d \xi=\frac{\alpha}{T} \int_{E_{m}}^{E}(E-\xi)^{3} e^{\frac{\xi-E_{0}}{T}} d \xi \\
& =\alpha\left\{6 T^{3} e^{\frac{E-E_{0}}{T}}-\left[\left(E-E_{m}\right)^{3}+3 T\left(E-E_{m}\right)^{2}+6 T^{2}\left(E-E_{m}\right)+6 T^{3}\right] e^{\frac{E_{m}-E_{0}}{T}}\right\} ;
\end{aligned}
$$




$$
\begin{aligned}
& \begin{array}{l}
\text { for } E_{-}<E<E: \\
\int_{E_{L}}^{E}(E-\xi)^{3} \rho(\xi) d \xi=\frac{\alpha}{T} \int_{E_{m}}^{E_{x} x}(E-\xi)^{3} e^{\frac{\xi-E_{0}}{T}} d \xi+b \int_{E_{\chi}}^{E} \frac{(E-\xi)^{3}}{\left(\xi-U_{0}\right)^{3 / 2}} e^{2 \sqrt{a\left(\xi-U_{0}\right)}} d \xi
\end{array} \\
& \left.=\alpha\left\{\left[(E-\xi)^{3}+3 T(E-\xi)^{2}+6 T^{2}\left(E-\frac{k}{\xi}\right)+6 T^{3}\right] \cdot \frac{\xi-E_{0}}{T}\right\}_{\xi}\right\}^{k}=E_{x}-2 b\left\{\left(\frac { 1 } { ( 2 \sqrt { a } ) ^ { 5 } } \left[(2 \sqrt{a} x)^{4}\right.\right.\right. \\
& \left.-4(2 \sqrt{a} x)^{3}+12(2 \sqrt{2} x)^{2}-24(2 \sqrt{a} x)+24\right]-\frac{3 c}{(2 \sqrt{2} x)^{3}}\left[(2 \sqrt{a} x)^{2}-2(2 \sqrt{2} x)+2\right] \\
& \left.+\frac{3 c^{2}}{2 \sqrt{a}}+\frac{c^{3}}{x}\right) \cdot 2 \sqrt{a} x-2 \sqrt{a} c^{3}\left[\ln x+(2 \sqrt{a} x)+\frac{(2 \sqrt{a} x)^{2}}{2.2 !}+\frac{(2 \sqrt{a} x)^{3}}{3.3 !}\right. \\
& \left.\left.+\ldots \frac{(2 \sqrt{2} x)^{n}}{n \cdot n !}+\frac{(2 \sqrt{a} x)^{n}}{n \cdot n !} \frac{2 \sqrt{a} x}{(n+1)^{2}}+\ldots\right]\right\} \begin{array}{l}
x=\sqrt{E-U_{0}} \\
x=\sqrt{E_{x}-U_{0}}
\end{array}
\end{aligned}
$$

The sories appearing in the above expressions were terminated when the ratio of the last term to the sum of the preceding terms is $10^{-12}$.

A third option (density indicator IROE $=2$ ) has also been addod for the nuclear-level density function. If the spin-dependence parameter is treated ae being independent of energy or if the total level density including levels degenerate in the magnetic quantum number is considered, the Gilbert and Camoron composite nuclear-level density formula can be represented by

$$
\rho(E)= \begin{cases}\frac{\alpha}{T} e^{\frac{E-E_{0}}{T}} & E \leq E_{X} \geq E_{m} \\ \beta\left(E-U_{0}\right)-\frac{5}{4} e^{2 \sqrt{a\left(E-U_{0}\right)}} & E>E \geq E_{m}\end{cases}
$$

and $\beta$ is a new input quantity. 
For $E_{x} \geq \epsilon \geq E_{m}$ the integral that appears in the boundary condition remains the same, viz.

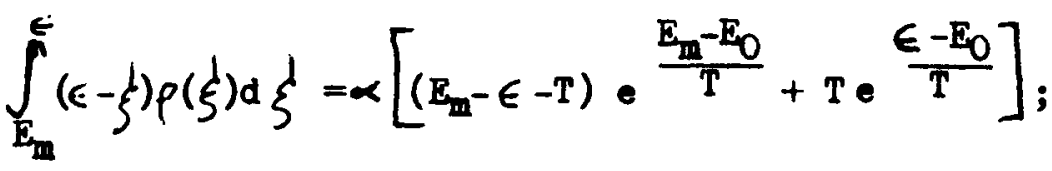

for $E_{m} \leq E_{x}<\epsilon:$

$\int_{E_{m}}^{\epsilon}(\epsilon-\xi) \rho(\xi) d \xi=\alpha\left[\left(E_{m}-\epsilon-T\right) e^{\frac{E_{m}-E_{0}}{T}}+(\epsilon-E x+T) e \frac{E_{\lambda}-E_{0}}{T}\right]$

$+\beta\left\{\left[\frac{4 M^{4}}{N}+\frac{N}{\sqrt{a}}\right] e^{2 \sqrt{a} N^{2}}-\left[4 M^{3}+\frac{M}{\sqrt{a}}\right] e^{2 \sqrt{a} M^{2}}+\left[16 \sqrt{a} M^{4}+\frac{1}{\sqrt{a}}\right] \int_{N}^{M} e^{2} \sqrt{a} \xi^{2} d \xi\right\}$

where $M \equiv\left(\epsilon-U_{0}\right)^{\frac{1}{4}}$ and $N \equiv\left(E_{X}-U_{0}\right)^{\frac{1}{4}}$.

Similarly, for $E \geq E \geq E_{\text {m }}$ the integral that appears in the normalization factor remains the same,

$\int_{E_{m}}^{E}\left(E-\frac{1}{\xi}\right)^{3} \rho(\xi) d \xi=\alpha\left\{6 T^{3} e^{\frac{E-E_{0}}{T}}-\left[\left(E-E_{m}\right)^{3}+3 T\left(E-E_{m}\right)^{2}+6 T^{2}\left(E-E_{m}\right)+6 T^{3}\right] e^{\frac{E_{m}-E_{0}}{T}}\right\} ;$

for $E_{m} \leq E_{\chi}<E$ :

$\int_{E_{m}}^{E}(E-\xi)^{3} \rho(\xi) d \xi=\alpha\left\{\left[(E-\xi)^{3}+3 T(E-\xi)^{2}+6 T^{2}(E-\xi)+6 T^{3}\right] \cdot \frac{\xi-E_{0}}{T}\right\}_{E_{m}}^{E_{x}}$

$-\beta\left[\left(\frac{\frac{1}{9} 9}{\sqrt{a}}-\frac{9 \xi^{7}}{4 a}+\left(\frac{63}{16 a}-3 c\right) \frac{\xi^{5}}{\sqrt{a}}-\left(\frac{63}{16 a}-3 c\right) \frac{5 \xi^{3}}{4 a}+\left[\left(\frac{63}{16 a}-3 c\right) \frac{15}{16 a}+3 c^{2}\right] \frac{\frac{f}{\sqrt{a}}}{\sqrt{2}}\right.\right.$

$\left.\left.+\frac{4 c^{3}}{\xi}\right\} e^{2 \sqrt{a} \xi^{2}}\right]_{N}^{L}+4 \beta\left\{\left[\left(\frac{63}{16 a}-3 c\right) \frac{15}{16 a}+3 c^{2}\right] \frac{1}{4 \sqrt{a}}+4 c^{3} \sqrt{a}\right\} \int_{N}^{L} e^{2 \sqrt{a} \xi^{2}} d \xi$, 
where $\mathrm{L} \equiv\left(E-U_{0}\right)^{\frac{1}{4}} \quad N \equiv\left(E_{X}-U_{0}\right)^{\frac{1}{4}}$ and $C \equiv E-U_{0}$

The integrals appearing in the above expressions were obtained numoricalls using Simpson's Rule.

1.4 PRDMARY TRANSITION PROBABILITI OPTIONS

In the original CASCADE there is an option that allows the primary transition probabilities to be input when thermal neutron capture is being treated. There has been no provision for a similar treatment for the enorgetic neutron capture process. However, direct neutron capture ${ }^{(3)}$ mas occur, and this results in an excess of high energy $\gamma$-rajs for the primary transitions. This has been observed particularly in the mass regions $A \approx 200$ and $A \approx 60$. To allow for this effect, it would be useful in a few cases were data is arailable to be able to input the primary transition probabilities for energetic neutron capture. In order to achieve this without modifying the program, the following steps may be carried out:

a) For each neutron energy or for each neutron group, the primary transition probabilities may be input and a separate CASCADE calculation carried out just as for thermal capture. The problems may be stacked, and the outputs written on a single tape.

b) A CASING calculation is carried out for each CASCADE calculation. All of the required information is then arailable, but not in the proper format for input into our design codes. A small program, REPUN, takes the output data from CASING and repunches it in the proper format, 1.e., the same format as the output from a normal CASCADE-CASING group calculation. 
(W) Astronuclear

c) If one wishes to collapse the neutron group structure, CASING has been modified to accept the repunched cards as input and will carry out the required group collapsing. 


\subsection{GAMMA RAY PRODUCTION CROSS SECTIONS FROM CASCADE-CASING;}

INELASTIC SCATTERING OF NEUTRONS FROM VARIOUS ELEMENTS

\section{$2.1 \quad 27 \mathrm{Al}$}

The energies and branching ratios for the nuclear levels of ${ }^{27} \mathrm{Al}$ are shown in Figure 2-1. The nuclear levels were obtained from references 4, 5, and 6; the branching ratios were obtained from ref. 4 except for the $3.951 \mathrm{MeV}$ level which is unknown. It is assumed that transitions from this level $\left(\frac{7}{2}^{+}\right)$ occur to the ground state $\left(\frac{5}{2}^{+}\right)$. Gilbert and Cameron's composite nuclear leveldensity formula $($ IROE $=3$ ) was selected, and the parameters required in the density expression were obtained from ref. 2. The values of the parameters are: $a=3.45 \mathrm{MeV}^{-1}, b=.0392 \mathrm{MeV}^{-1}, E_{X}=9.6 \mathrm{MeV}, E_{0}=-0.35 \mathrm{MeV}, U_{0}=1.8 \mathrm{MeV}$, and $\mathrm{T}=2.08 \mathrm{MeV}$.

The initial excitation functions for the discrete levels were input for the entire neutron energy range of interest, i.e. $\mathrm{E}_{\mathrm{a}}=10 \mathrm{MeV}$. From 4.19 $\mathrm{MeV}$ to $8.56 \mathrm{MeV}$ the experimental data of Kinney and Perey ${ }^{(7)}$ were used. Outside of this energy range the experimental data were complemented by calculated values obtained with the HELENE code ${ }^{(8)}$. The HEIENE code is based on the Hauser-Feshbach model and yields cross sections for reactions which proceed through a compound nucleus. The optical model parameters for the HELENE calculation were obtained from ref. 9. Some of the experimental data(7) for the excitation functions are for the excitation of more than one level. Upon comparing the sum of the calculated values of the excitation cross section for the $0.843 \mathrm{MeV}$ and 1.013 $\mathrm{MeV}$ levels, it was found to agree very well with the experimental results. For some of the higher energy levels, the experimental results tend to be lower than the theoretical. If the experimental data corresponded to the excitation of 
more than one level, the sum of the excitation cross sections for these levels was made equal to the experimental results, but the ratios of the cross sections for the individual excitation cross sections were obtained from the HELENE results. Additional calculated values of the excitation functions over various portions of the neutron energy range are given in refs. 9 (which is used in the ENDF and is taken from ref. 10), 11 and 12. Further experimental results are given in refs. 4,5 , and 13.

The inelastic cross section was obtained from experimental data given in BNI-325 $\left(\mathcal{U}_{4}\right)$ and the formula,

$$
\sigma_{\text {in }}=\sigma_{n e}-\sigma_{n p}-\sigma_{n \alpha}-\sigma_{n \gamma} .
$$

The HELENE results for the inelastic cross section agreed very well with the cross section obtained from the experimental data.

Table I gives the group boundaries for 12 neutron energy groups and 13 gamma energy groups. The program CASCADE has been run for a number of elements to yield garma-ray spectra for each of the 12 neutron groups. Subsequently, the CASING code has been run to produce the various $12 \times 13$ matrices whose elements are essentially the gamma-ray production cross sections. These matrices are described in ref. 1. Table IIa gives the radiant energy (MeV) emitted per inelastic scattering event for $n+{ }^{27} \mathrm{Al}$. Henceforth this will be referred to as the $Q(n, \gamma)$ matrix. Also shown in the table are values that have recently become available ${ }^{+}$at WANL for use in the transport codes. This data is based on an ORNL compllation by Ford ${ }^{(15)}$. The standard number of broad neutron groups used in the output of the APPROPOS code over the energy range .015 MeV to $10 \mathrm{MeV}$ is six. The upper boundaries of the groups in $\mathrm{MeV}$ are: $10,2.23, .821,0.388,0.111$, and 0.0409 . Note that each of the boundaries is equal to one of the boundaries + Provided by R. Soltesz 
of the 12 groups given in Table I. Using a neutron flux that represents an average flux in the BATH shield, the $12 \times 13$ matrix has been collapsed to a $6 \times 13$ matrix. These data are shown in Table IIIa for 27 Al. Also shown is the matrix that has been used in the POINT or APPROPOS library.

When most of the following calculations were carried out, the new options described in Part 1 were not available.

$2.2 \quad 56 \mathrm{Fe}$

The nuclear levels and branching ratios for $56_{\mathrm{Fe}}$ are given in Figure 2-2. They were obtained from refs. 16, 17, 18, 19,20,21, and 22. Transitions from the $3.388\left(6^{+}\right)$MeV level are unknown; we have assumed the nucleus decays to the $3.122\left(5^{-}\right) \mathrm{MeV}$ level.

The nuclear level density based on the Fermi gas model of the nucleus was used, i.e. IROE $=1$. To determine the parameters for the density, $\rho$, a knowledge of the density at two energies is required. By counting observed levels of both parities in an energy interval about $4 \mathrm{MeV}$ (see references 16, 17 , and 21) it is found that

$$
\rho \approx 12 \text { levels } / \mathrm{MeV} \text { at } \mathrm{E} \approx 4 \mathrm{MeV} \text {. }
$$

In ref. 23 a level density (including both parities) of $\rho=(560 \pm 280)$ levels $/ \mathrm{MeV}$ is found for $E=10.3 \mathrm{MeV}$, where $E$ is the excitation energy as measured from the ground state. Using Gilbert and Cameron's formula and parameters (2) for the level density gives $P=1200$ levels/MeV at $E=10.3 \mathrm{MeV}$. It has been assumed for this calculation that:

$$
P=800 \text { levels/MeV at } 10.3 \mathrm{MeV} \text {, }
$$

and the parameters $a$ and $b$ are found to be:

$$
a=3 \mathrm{MeV}^{-1}, \quad b=1.2 \times 10^{-2} \mathrm{MeV}^{-1} \text {. }
$$


The relative level density $P(10 \mathrm{MeV}) / P(4 \mathrm{MeV})$ agrees $f a i r l y$ well whth an experimental value given in ref. 24 .

The inelastic cross sections for exciting the various levels have

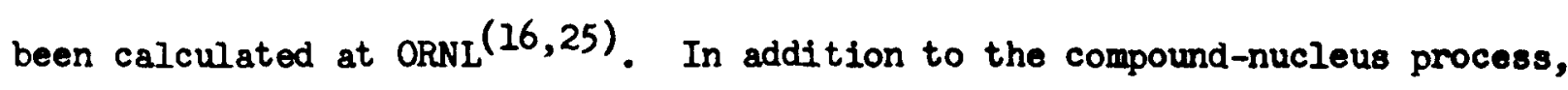
the inelastic scattering resulting in the excitation of the $0.846 \mathrm{MeV}$ level includes the direct interaction excitation. Experimental data is found in refs. 20 and 25. The excitation functions were input only for $E \leq E_{m}$.

Tables IIb and IIIb give the $Q(n, \gamma)$ matrices for 56 Fe.

2.3 $48 \mathrm{Ti}$

The input required for this calculation was given in ref. 1. The radiant energy emitted per inelastic event is given in the form of the $Q(n, \gamma)$ matrices in Tables IIc and IIIc.

2.4 INELASTIC NEUTRON SCATTERING IN Pb

$2.4 .1208_{\mathrm{Pb}}$ *

Natural lead consists of $52 \%{ }^{208} \mathrm{~Pb}$. The nuclear level scheme and the branching ratios for ${ }^{208} \mathrm{~Pb}$ were taken from the "Table of Isotopes" (26) and are shown in Figure 2-3a. The continuum is assumed to start at $E_{m}=4.15 \mathrm{MeV}$, with the density of levels given by the Fermi gas expression:

$$
\rho(E)=6 e^{2 \sqrt{a E}} ; E \geq E_{m}
$$

The parameters $\underline{a}$ and $\underline{b}$ are evaluated from the densities at 4.15 and $7.4 \mathrm{MeV}$, the latter energy representing the binding energy of a neutron in $208 \mathrm{~Pb}$.

By counting levels(27) of both parities, it is found that

$$
\rho \simeq 9 \frac{\text { levels }}{\mathrm{MeV}} \text { at } 4.15 \mathrm{MeV}
$$

* The calculation of inelastic neutron scattering in $208 \mathrm{~Pb}$ was carried out by S. Fody. 
The level density at the binding energy is estimated, from the observed (2) s-wave neutron resonance density of $20 \mathrm{MeV}^{-1}$ for ${ }^{207} \mathrm{~Pb}$, in the following manner. Since the ground state of ${ }^{207} \mathrm{~Pb}$ has spin and parity of $I^{\pi}=1 / 2^{-}$, the low-energy s-wave neutron resonances will excite only those ${ }^{208} \mathrm{~Pb}$ states which have $\mathrm{J}^{\pi}=\mathrm{O}^{-}, \mathrm{I}^{-}$. Using the density function $(2)$

$$
\frac{2 J+1}{2 \sigma^{2}} e^{-\frac{\left(J+\frac{1}{2}\right)^{2}}{2 \sigma^{2}}}
$$

for the angular-momentum dependence of the observed level density, it is found that $2.4 \%$ of the ${ }^{208} \mathrm{~Pb}$ levels have $\mathrm{J}=0$, and $6.7 \%$ have $\mathrm{J}=1$. (The spin dependence parameter $\sigma$ is taken to be 4.55 , the same as for ${ }^{207} \mathrm{~Pb}$ ). Assuming that levels of positive and negative parity are equal in numbers, one obtains that

$$
\rho \simeq 20 \times \frac{1}{0.091} \times 2=440 \frac{\text { levels }}{\mathrm{MeV}} \text { at } 7.4 \mathrm{MeV}
$$

These two densities and the expression for the level density yield the level density parameters of

$$
a=14 \mathrm{MeV}^{-1}
$$

and

$$
\mathrm{b}=1.37 \times 10^{-6} \mathrm{MeV}^{-1}
$$

which are used in running CASCADE.

In the CASCADE run the standard twelve neutron groups were treated, using the option S-I for groups above $E_{m}$ and the option $S-I_{0}-2$ for groups below $E_{m}$. The required inelastic cross sections for exciting the levels below $E_{m}$ were based on the data obtained by Towle and Gllboy $(28,29)$. When collapsing, in CASING, the twelve neutron groups into six coarse groups, the required average 
inelastic scattering cross sections for the twelve groups were estimated from the inelastic cross section for natural lead, as given (30) in ref. 30 . $2.4 .2 \quad 207 \mathrm{~Pb}$

$207 \mathrm{~Pb}$, which is $22 \%$ abundant in natural lead, is simply doubly magic ${ }^{208} \mathrm{~Pb}$ minus one neutron, and hence low-lying $207 \mathrm{~Pb}$ levels should be mainly neutron hole states. By virtue of its closeness to ${ }^{208} \mathrm{~Pb}$, ${ }^{207} \mathrm{~Pb}$ is a muchstudied nucleus. Five levels below $2.9 \mathrm{MeV}$ are known to be purely of the neutron hole configuration, and three others are known to be core excitations. Another level in this region was seen by one experimenter only (31), and was omitted in this analysis since no spin assignment has been given. Above 2.9 $\mathrm{MeV}$ many levels have been seen but few have spin assignments. Hence for purposes of this analysis, the lower boundary of the continuum was taken at $2.9 \mathrm{MeV}$.

The number of levels in the $1 \mathrm{MeV}$ interval immediately above $2.9 \mathrm{MeV}$ was gotten from the works of Moyer et al ${ }^{(31)}$, Nellis ${ }^{(32)}$ and Vallois et al(33). The first mentioned experiment sees many more levels than the other two, but only those levels that appear unambiguously in this interval in their published $\left(d_{,} d^{\prime}\right)$ spectrum ( 27 in all) were included by us. Above the neutron binding energy (6.737 $\mathrm{MeV}$ ) Macklin et al ${ }^{(34)}$ give the density of $l=0$ and $l=1$ resonances in the ${ }^{206} \mathrm{~Pb}$ $+\mathrm{n}$ reaction. Although the theoretical ratio of $l=0$ to $l=1$ spacing is approximately 3, Macklin et al. find this ratio to be 6.7 . It was felt that for the present analysis, the most reasonable approach was to lump all resonances together, as is done for ${ }^{206} \mathrm{~Pb}$, and thus facilitate determination of the density parameters $a$ and $b$ for input to CASCADE. Instead of Macklin's determination that $\rho(l=0)=133$ levels $/ \mathrm{MeV}$ and $\rho(l=1)=20$ levels/MeV, the present calculation 
started from the combined density of $l=0$ and $l=1$ ( or $\mathrm{J}^{\pi}=1 / 2^{+}, 1 / 2^{-}, 3 / 2^{-}$) levels as $153 / \mathrm{MoV}$ to obtain $a$ and $b: 6.018 \mathrm{MeV}^{-1}$ and $3.18 \times 10^{-3} \mathrm{MeV}^{-1}$ rospectively.

Electromagnetic transitions between low-lying states of ${ }^{207} \mathrm{~Pb}$ have been analyzed recently by Nellis(32). His results are more extensive than, but generally consistent with, earlier investigations of Alburger and Sunjar(35). Two more relatively weak transitions were postulated for the present work between those levels whose known structure suggests that a transition between them would go readily, but where no transitions have yet been observed. A few branching ratios, not given in the ilterature, were also guessed at based on the Moszkowski(36) model. (See Appendix II). The final decas scheme is shown in Figure 2-3b.

This section will be concerned with excitation probabilities for low-lying levels in inelastic scattering. These were obtained 1) using the CASCADE model, $r_{i}^{0}=$ initial excitation probability $=\left(\epsilon-E_{i}\right) / \sum\left(\epsilon-E_{j}\right)$; and 2) from the results of an inelastic scattering experiment of Cranberg et al (37) coupled to calculations of the code HELENE $(8)$ where necessary. The two calculations were compared with each other and against experimental cross sections for Individual gama-rays.

Cranberg gives excitation functions for five levels below $2.8 \mathrm{MeV}$ in excitation at neutron energies from 1.7 to $5 \mathrm{MeV}$, and for one of these levels at 7 and $8 \mathrm{MeV}$. One of his "levels" appears to be a $2.6-2.7 \mathrm{MeV}$ triplet, thus accounting for all seven of the excited states included in our analysis. The experimental cross section to this "level" was divided among the three members of the triplet in proportion to each one's share of the inelastic 
neutron strength in Cranberg's $3.5 \mathrm{MeV}$ spectrum. For CASCADE input, the assumption was made that all level strengths decay slowly with increasing energy as does the $2.7 \mathrm{MeV}$ triplet, the "level" to which the inelastic cross section was measured up to $8 \mathrm{MeV}$. This is in agreement with HELENE calculations. Excitation functions for the .57 and $.89 \mathrm{MeV}$ states below $2 \mathrm{MeV}$ were calculated by HELENE. The calculation included Porter-Thomas width fluctuations and considered competition from neutron capture, although the effects of capture competition were negligible. The optical parameters for neutrons on ${ }^{207} \mathrm{~Pb}$ were taken from the work of Moore (38), who did an analysis of neutron scattering from ${ }^{207} \mathrm{~Pb}$ at $0.5,1.0$ and $2.5 \mathrm{MeV}$. Parameters at the latter two energies were used in HELENE for inelastic cross section calculations at 1.0 and $2.0 \mathrm{MeV}$ respectively. At higher energies, the energy dependent optical parameters of Bechetti and Greenless (39) were used. These parameters are listed in Table IV. At 2 MeV HEIENE gave a cross section of .43 barns to the $570 \mathrm{keV}$ level, and .28 barns to the $894 \mathrm{keV}$ level. Cranberg's experimental results at $50^{\circ}$ multiplied by 4 were .51 and .40 barns respectively. Therefore HELENE calculations at 1 and 2 MeV were increased by about $30 \%$ to agree with experiment. Similarly, above $5 \mathrm{MeV}$ they were multiplied by 4 to match Cranberg's data at $5 \mathrm{MeV}$. Figure 2-4 shows the excitation probabilities (in barns) input to CASCADE.

It is possible to compare CASCADE results on the excitation functions of discrete gamma-rays with experimental data only if the total inelastic scattering cross section of ${ }^{207} \mathrm{~Pb}$ is known. Unfortunately, the only available inelastic cross sections are for natural lead and are quite old. Hence significance of any comparison is diminished. There are two cross section 
sources: the Atomic Weapons Research Establishment Library ${ }^{(30)}$ and the GAM library. They are in rough agreement except above $7 \mathrm{MeV}$, where the GAM cross section is flat, but the AWRE cross section drops from 2.4 to .4 barns at 10 MeV. The two sources of experimental data are a Texas Nuclear Report $(40)$ and a conference abstract by a Los Alamos Group (4I). In the only region of overlap, the cross section for production of the $1.064 \mathrm{MeV}$ gamma ray, the former is lower than the latter by about $25 \%$.

Figure 2-5 shows the comparison between data and calculations. In general the calculations (CASCADE's $P\left(E_{\gamma}\right)$ multiplied by the inelastic cross sections) fall below the data, but this may be due to the use of the natural rather than isotopic inelastic cross sections. If the same operation applied to all three calculations (e.g., multiply by 2 above $3 \mathrm{MeV}$ ) brought them Into agreement with the data, then it would seem that use of natural cross sections was at fault, but if no operation could bring them into agreement, then the fault would lie either with the CASCADE calculation or with the data. From the figure it is seen that the only substantial disagreement between the calculations using CASCADE's excitation probabilities and "experimental" excitation probabilities is for the $1.064 \mathrm{MeV}$ gama ray. The experimental excitation probabilities give a shape somewhat closer to the actual energy variation of the data. Doubling both sets of calculations between 3 and $5 \mathrm{MeV}$ brings them into better agreement with the data, and the experimental excitation probability calculations also need to be multiplied by 1.5 below $3 \mathrm{MeV}$. The other calculations, though, are already in agreement below $3 \mathrm{MeV}$ for the $1.064 \mathrm{MeV}$ gamma ray, but are somewhat below the data for the other two. Hence no single operation could bring about good agreement for all three gamma rays. 
The conclusion is that there seems to be a slight preference for use of experimental excitation probabilities, but without a measurement of the isotopic inelastic cross section no definite conclusions are possible. The experimental excitation probability CASCADE has been used in the final library file, however, in view of its apparent better agreement with experimental ganma ray production data.

2.4.3 $206 \mathrm{~Pb}$ - Inelastic

${ }^{206} \mathrm{~Pb}$ is $\sim 25 \%$ abundant in natural lead. Spins and parities of 25 of its 31 known levels below $3.6 \mathrm{MeV}$ have been tentatively or positively determined, and many $\gamma$ decays between these levels are known from studies of ${ }^{206} \mathrm{Bi} \beta^{+}$decay. For this work $E_{m}$, the energy at which the level continuum begins, was taken to be $3.6 \mathrm{MeV}$. The most recent tentative assignments for levels below this energy were accepted as definite. The six levels below $3.6 \mathrm{MeV}$ which were lacking even tentative $\mathrm{J}^{\pi}$ assignments were omitted from consideration. Branching ratios for decays of levels not populated in the ${ }^{206} \mathrm{Bi}$ decay chain were calculated using the decay probabilities calculated by Moszkowski for single particle transitions, but retarded by the empirical retardation factors of Goldhaber \& Sunyar (42). (See Appendix II). Hopefully, use of the retardation factors complled by Goldhaber \& Sunyar from experimental strengths of transitions of each multipolarity minimized the hazards in this method. Agreement with experimental results for the decay of five ${ }^{206} \mathrm{~Pb}$ levels was fair for both the single particle and the retarded estimates. To calculate the level density parameters $a$ and $b$ in the Fermi gas expression used by CASCADE, level densities at two regions of excitation are needed. The level density from 3.6 to $4.6 \mathrm{MeV}$ was taken from Solf et al. (43) who show 25 levels in this interval. The level density at high 
excitations was gotten from the low onergy resonance density in the ${ }^{207} \mathrm{~Pb}$ $+n \rightarrow{ }^{208} \mathrm{~Pb}$ reaction, which gives the density of $0^{ \pm}, 1 \pm, 2^{+}$levels in ${ }^{208} \mathrm{~Pb}$ (assuming $l=0$ or 1 ). This was assumed to be identical to the density of these levels in $206 \mathrm{~Pb}$, since no capture experiments on ${ }^{205} \mathrm{~Pb}$ have been done. Use of the above level density formula near closed shells has been cautioned against ${ }^{(2)}$, but extraction of $a$ and $b$ from expariments increases the likelihood of Its giving satisfactory representation of the true level density. From the density of $0^{ \pm}, 1^{ \pm}, 2^{+}$levels one can find the total level density using the Bethe formula ${ }^{(2)}$ :

$$
\rho(E, J) \simeq \rho_{0}(E) \frac{1}{\sqrt{2 \pi} \sigma} \frac{2 J+1}{2 \sigma^{2}} \exp -\frac{\left(J+\frac{1}{2}\right)^{2}}{2 \sigma^{2}}
$$

where of 1s the "spin cut-off parameter" tabulated by Gilbert \& Cameron ${ }^{(2)}$, and $\frac{2 J+1}{2 \sigma^{2}} \exp \left[-\frac{\left(J+\frac{1}{2}\right)^{2}}{2 \sigma^{2}}\right]$ is the Iractional density for levels of spin J. The parameters $a$ and $b$ thus obtained were $a=4.81 \mathrm{MeV}^{-1}, b=3.47 \times 10^{-3} \mathrm{MeV}^{-1}$.

There are 11 levels between 0 and $150 \mathrm{keV}$ neutron energy in ${ }^{207} \mathrm{~Pb}+\mathrm{n}$. Bypassing difficulties of determining the spin of each one, they were considered as a group so that the total density was determined from

$$
P_{t t 1}(E)=\frac{11}{.15} \times 2 \sigma^{2}\left[0 \frac{-1}{8 \sigma^{2}}+3 e^{-9 / 8 \sigma^{2}}+\frac{5}{2} e^{-25 / 8 \sigma^{2}}\right]^{-1} .
$$

Excitations, spins and parities for 25 levels below $3.6 \mathrm{MeV}$ were gotten from several sources $(26,44,45)$, with considerable duplication among them. Transition probabilities were taken mainly from the work of Alburger and Price ${ }^{(46)}$ as revised by Herrlander ${ }^{(47)}$ from his own and other experiments. For those levels 
where no transitions have been seen, branching ratios were calculated as described above and further altered by bringing all extremes in towards $50 \%$. The level scheme and branching ratios are shown in Figure 2-3c.

The one remaining CASCADE input variable which must be gotten from the literature is the set of initial excitation probabilities for the discrete levels. Cranberg $(37)$ has measured these directly for 11 levels at neutron energies between 2.5 and $4.25 \mathrm{MeV}$. His values were input to CASCADE along with extrapolations outside his energy range and $\frac{\epsilon-E_{i}}{\sum_{i}\left(\epsilon-E_{i}\right)}$ variation (as is built into CASCADE as an option) for levels he didn't see, but the CASCADE results did not give good agreement with experiment, presumably because of poor extrapolation. The final results were gotten with the $\frac{\epsilon-E_{i}}{\sum_{i}\left(\epsilon-E_{i}\right)}$ variation for all 25 discrete levels, and agreement with experiment was better, as is shown below.

CASCADE results for the standard 12 group neutron energy mesh are shown in the printed and plotted output. CASCADE calculations can be compared with several experimental results in the literature: one for excitation functions of seven gamma-rays at neutron energies from 1.4 to $3 \mathrm{MeV}^{(48)}$, and one for the gamma spectrum at $E_{n}=5 \mathrm{MeV}^{(12)}$. Figure 2-6, from BNL-325, shows the energy variation for production of various gamma rays. The calculation where inftial excitation probabilities were calculated by CASCADE gives slightly better agreement with experiment than the calculation where some were taken from Cranberg $(37)$, although both are quite good. To normalize CASCADE results (relative excitation probabilities) for this comparison, they were multiplied at each neutron energy by the total inelastic cross section, taken to be just the sum of the individual inelastic cross sections given by Cranberg. 
The gamma spectrum from inelastic neutron scattering on ${ }^{206} \mathrm{~Pb}$ has been measured by Nellis et $a,(49)$ and CASCADE results (for $E=5.4 \mathrm{MeV}$ ) can be compared with his preliminary spectrum at $E_{n}=5 \mathrm{MeV}, \theta_{1 a b}=55^{\circ}$, if one assumes that the gamma rays are 1sotropic. Figure 2-7 shows this comparison, with CASCADE results normalized to equalize the experimental and predicted strength of the $.803 \mathrm{MeV}$ peak. Agreement between the two is fair-to-good especially when one averages over gamma energy groups. CASCADE predicts much less continuum than is seen by Nellis, even though the CASCADE output plot seems to indicate otherwise. It must be remembered that for D-D gamma rays, CASCADE gives the peak height as the number of gammas per inelastic ovent, but for continuum gammas, CASCADE gives probability/MeV/inelastic event of getting a gamma at each energy. Selecting $d E \gamma$ as four times the channel width of Nellis (the approximate FWHM of his peaks) gives a small continuum, thus in Figure 2-7 CASCADE results are plotted above the experimental continuum.

A small program, FRAC, was written so that $Q(n, \gamma)$ matrices for natural $\mathrm{Pb}$ could be generated. Each matrix element for the composite material is obtained from:

$$
Q=\sum_{i} \sigma_{i} f_{i} Q_{i} / \sum_{j} \sigma_{j} f_{j}
$$

where $f_{i}$ is the abundance of the $i^{\text {th }}$ isotope

$\sigma_{i}$ is the cross section for inelastic scattering from the $i^{\text {th }}$ isotope, and $Q_{i}$ is the radiant energy emitted per inelastic scattering event in the $i^{\text {th }}$ isotope.

The results for natural $\mathrm{Pb}$ are given in Tables IId, and IIId. 


\subsection{GAMMA-RAY PRODUCTION MATRICES FROM CASCADE-CASING;}

RADIATIVE CAPTURE OF NEUTRONS BY VARIOUS ISOTOPES

3.1

${ }^{48} \mathrm{Ti}(\mathrm{n}, \gamma){ }^{49} \mathrm{Ti}$

The level scheme ${ }^{(50)}$ and branching ratios for ${ }^{49} \mathrm{TI}$ are shown in

Figure 3-1. The branching ratios were obtained from the TRANSIT code (Appendix II), with some weighing based on observed spectra, e.g. see ref. 50. For thermal neutron capture, primary transition probabilities found in Nuclear Data ${ }^{(51)}$ were input. Gilbert and Cameron's composite nuclear level density formula was chosen (IROE $=3)$, and the required parameters were obtained from their paper, ref. 2. The results for thermal neutron capture are given in Table V, and compared with S. Fody's ${ }^{(52)}$ compilation based on Rasmussen's data ${ }^{(53)}$ and also the data presently in the POINT library.

The total $Q(n, \gamma)$ matrix for 12 neutron groups is given in Table VI. $3.2 \quad{ }^{206} \mathrm{~Pb}(n, \gamma){ }^{207} \mathrm{~Pb}$

The ${ }^{207} \mathrm{~Pb}$ level scheme and gamma cascade have been discussed in the section on inelastic scattering from ${ }^{207} \mathrm{~Pb}$.

${ }^{206} \mathrm{~Pb}$ is one of a very few nucleii to have had relative probabilities for orimary transitions to low-lying levels in $(n, \gamma)$ measured as a function of neutron energy below $10 \mathrm{MeV}$. Hence it was thought desirable to use experimental values of these probabilities in CASCADE rather than rely on the built in $\left(\epsilon-E_{i}\right)^{3}\left[\sum_{j}\left(\epsilon-E_{i}\right)^{3}+\int_{E_{m}}^{\epsilon}\left(\epsilon-E^{\prime}\right)^{3} \rho\left(E^{\prime}\right) d E^{\prime}\right]^{-1}$ model.

Biggerstaff et al $(54)$ have measured the relative strength of five gamma rays leading to low levels at five resonant energies between 16 and $75 \mathrm{kev}$. Folding the ${ }^{206} \mathrm{~Pb}$ total capture cross section of Macklin et al $(34)$ between 14 
and $60 \mathrm{keV}$ into these strengths yields (with some interpolation) the energy variation of direct excitation of four of the eight lowest levels of $207 \mathrm{~Pb}$ for the two lowest neutron energies in the standard twelve group mesh. It was necessary to make the assumption that $\sim 90 \%$ of the capture cross section leads to these levels. This is supported qualitatively by statements of Biggerstaff $(54)$ and Bergqvist (55). Direct excitation strengths for the remaining four levels were guessed at from their strengths in published spectra (which give upper limits at best) and the general shape of the excitation function for known levels. Bergqvist et al. measured high energy gamma rays from neutron capture in ${ }^{206} \mathrm{~Pb}$ (and thus the direct excitation of low-lying ${ }^{207} \mathrm{~Pb}$ levels) from 1.5 to 8.5 MeV neutron energy. Once again some extrapolation and guessing at weak levels was necessary to obtain direct excitation cross sections for the eight levels down to $6 \mathrm{MeV}$ and for four of these down to $1.5 \mathrm{MeV}$.

Between the neutron energies of Bergqvist and Biggerstaff some more interpolation was necessary. Although the Troubetzkoy model uses an energy variation of $\left(\epsilon-E_{i}\right)^{3} / \sum_{j}\left(\epsilon-E_{j}\right)^{3}$, he admits that this is the weakest part of his derivation. Furthermore it would be impossible to connect the two data sets with this function. Therefore the connection was made using a variation of $\sim E^{-1}$, which passed through the data at high and low energies. Table VII gives the excitation probabilities for the twelve $E_{n}$ of the standard neutron energy mesh and the eight ${ }^{207} \mathrm{~Pb}$ levels being considered. The sum of the probabilities for each neutron energy is 0.9 , as explained above. Figure 3-2 shows these cross sections in millibarns.

The complete calculation was carried out with a version II of CASCADE since CASCADE I does not allow any variation of direct transition probabilities 
with neutron energy. Unfortunately, no experimental data on the complete capture $\gamma$-spectrum of ${ }^{206} \mathrm{~Pb}$ could be found for verification of CASCADE results, but our lowest energy $\left(E_{n}=28 \mathrm{keV}\right)$ gamma spectrum does look qualitatively like those of Biggerstaff in this neutron energy region (remembering that in the CASCADE plots the continuum gamma probability is exaggerated with respect to discrete gamma rays). The total $Q(n, \gamma)$ matrix for 12 neutron groups is given in Table VIII. 3.3 $207 \mathrm{~Pb}-\mathrm{n}$ Capture

The gamma-ray spectrum resulting from neutron capture on ${ }^{207} \mathrm{~Pb}$ was calculated with a different ${ }^{208} \mathrm{~Pb}$ level scheme from the one used for inelastic scattering on ${ }^{208} \mathrm{~Pb}$. The new level scheme and branching ratios were taken from Nuclear Data Sheets $(56)$. This level scheme comprises 33 states below $6.3 \mathrm{MeV}$ in excitation rather than the 6 states below $4.15 \mathrm{MeV}$ used in the inelastic scattering analysis. The new analysis uses all levels and transitions, both certain and uncertain, given in Nuclear Data Sheets. All branching ratios are used as given in Nuclear Data Sheets.

Results of Initial CASCADE calculations were compared with experimental $\boldsymbol{\gamma}$-spectra of Biggerstaff et al ${ }^{(54)}$ taken at 20,30 and $45 \mathrm{keV}$ neutron energy. It was found that the transitions from the capturing state to the ground and first excited state were predicted much too small by the $(\epsilon-E i)^{3}(\epsilon)$ model in CASCADE. Hence these two transitions were enhanced at the expense of transitions to higher states by entering all initial transitions explicitly. Table IX gives a few transition probabllities as calculated by CASCADE and as entered explicitly. 
Although at $\sim 30 \mathrm{keV}$ neutron energy no change is larger than a factor of two, at $E_{n}=8 \mathrm{MeV}$, the CASCADE-calculated transition probabilities are at least two orders of magnitude smaller than the input values. The great enhancement in the input values reflects the likelihood of direct and semidirect capture to low lying states which has been seen to be important in this region (55). It greatly increases the amount of radiant energy assigned to high energy gammas. 


\subsection{COMMENTS ON THE RESULTS}

Comparisons of the data that have been used in the APPROPOS code for NERVA calculations with the CASCADE results and also the ORNL data as obtained from the POPOP 4 library can be made. Although there is no consistent pattern to the discrepancies for the inelastic scattering data, it is evident that in general the secondary gamma-ray production cross sections in the APPROPOS code should be updated and improved.

Because the binding energy of the neutron is available for excitation of the compound nucleus when radiative capture occurs, CASCADE results show that a significant percentage of the radiant energy can be released in gamma-rays of energy greater than $9.5 \mathrm{MeV}$. This fact has been ignored in the APPROPOS calculations. Although the capture cross sections tend to be small at high neutron energies, the most penetrating gammas are the ones being neglected. Shielding and heating calculations should be made to determine if this is a serious omission. 
(W. Astronuclear

5.0 FIGURES

5-1 


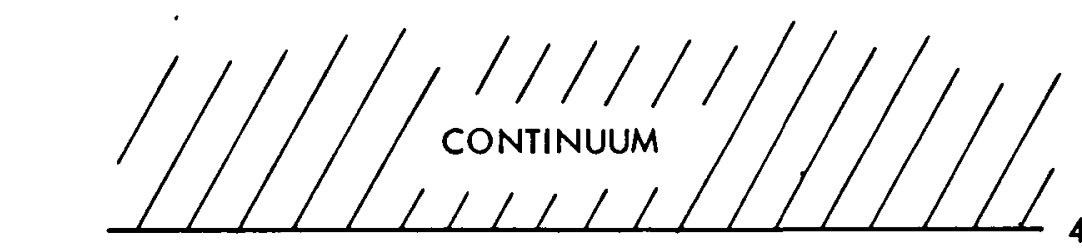

4.4

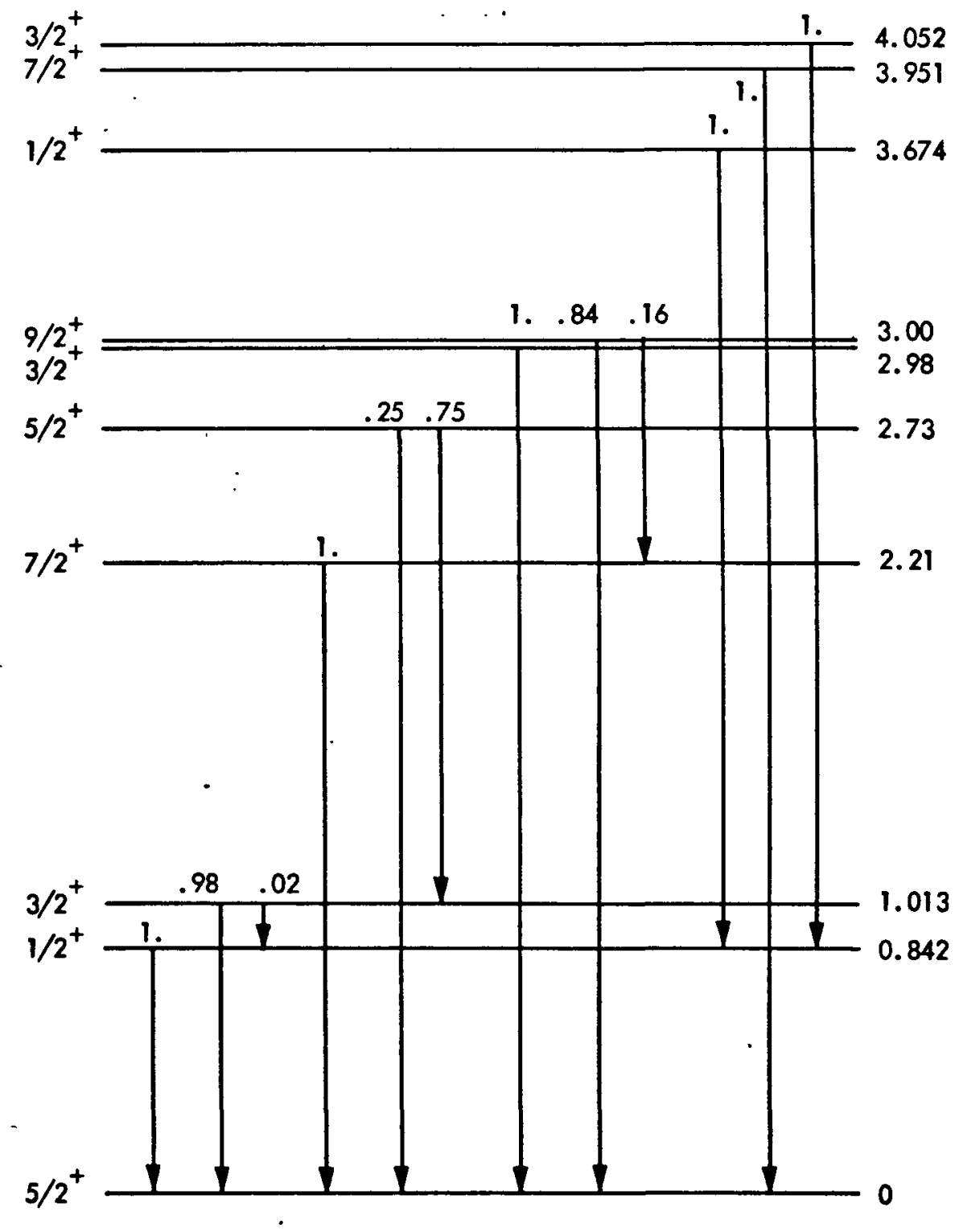

Figure 2-1. Energies and Branching Ratios for $10(n=9)$ Nuclear Levels of 27Al. A Continuum is Assumed for $E \geq 4.4 \mathrm{MeV}$ 
(W) Astronuclear

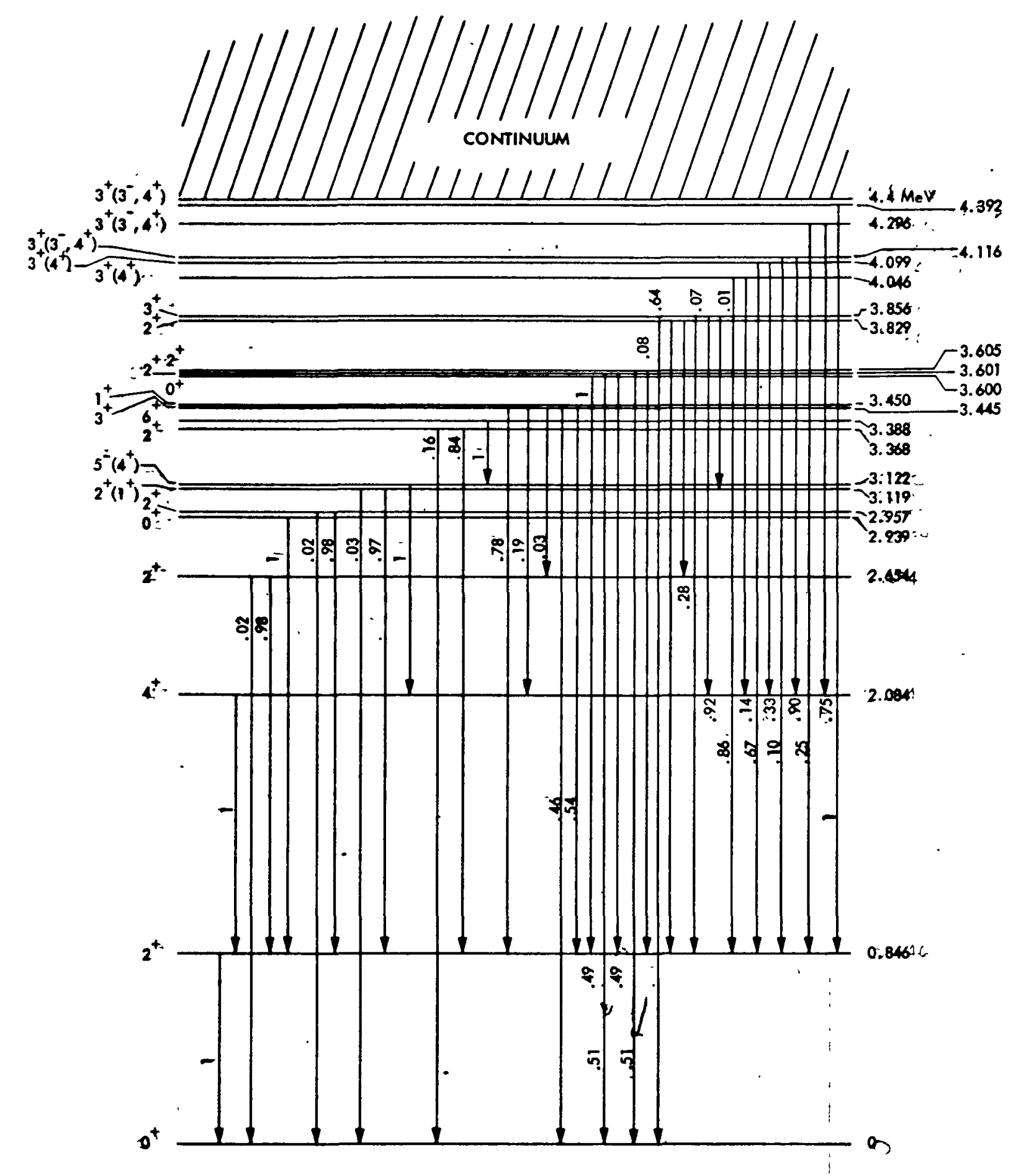

Figure 2-2. ${ }^{56}$ Fe Level Diagram with Gamma-Ray Branching Ratios for 22 $(n=21)$ Nuclear Levels. A Continuum is Assumed for $E \geq 4.4 \mathrm{MeV}$ 


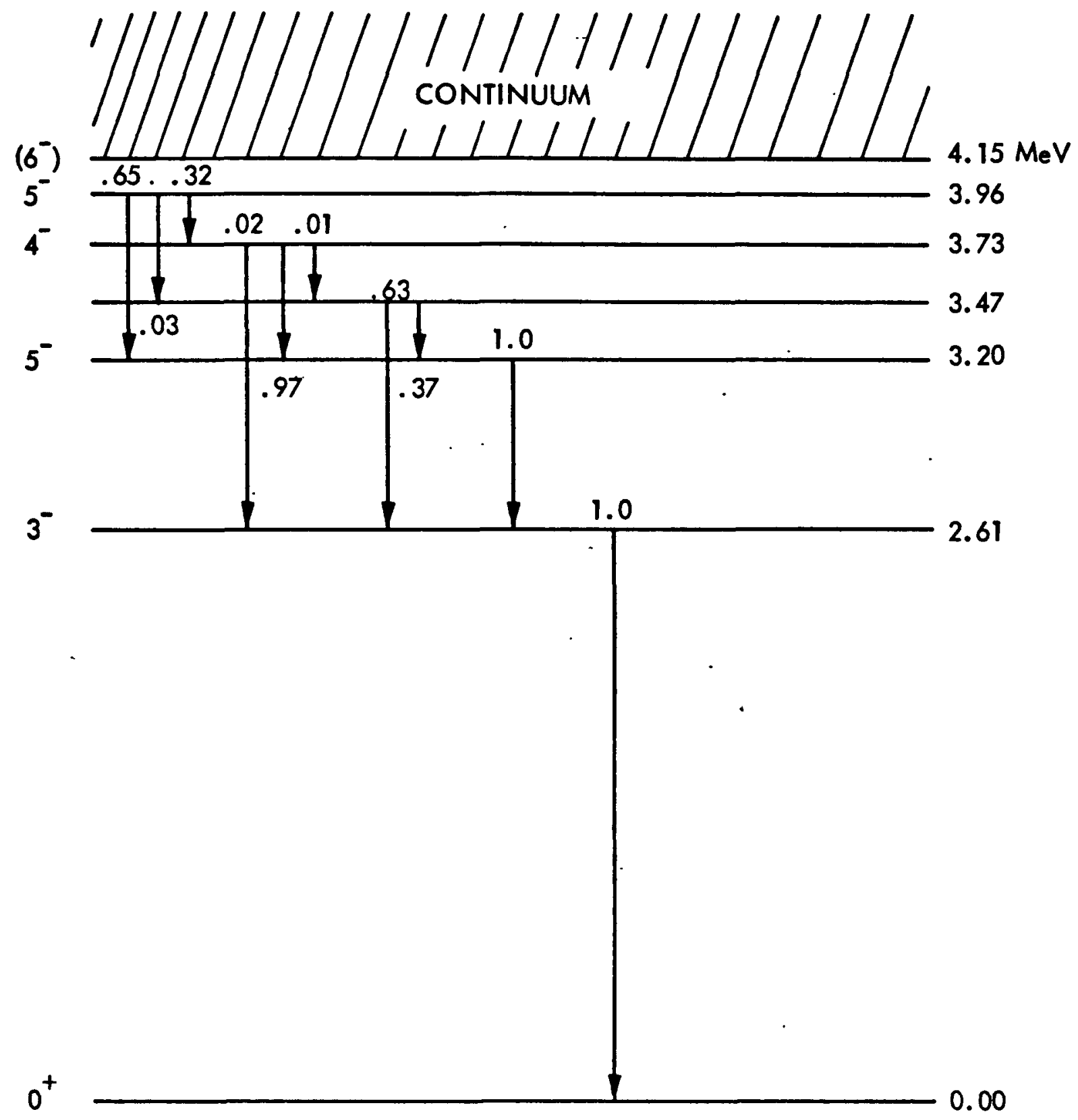

Figure 2-3a. Nuclear Level Scheme and Branching Ratios for $\mathrm{Pb}^{208}$ 


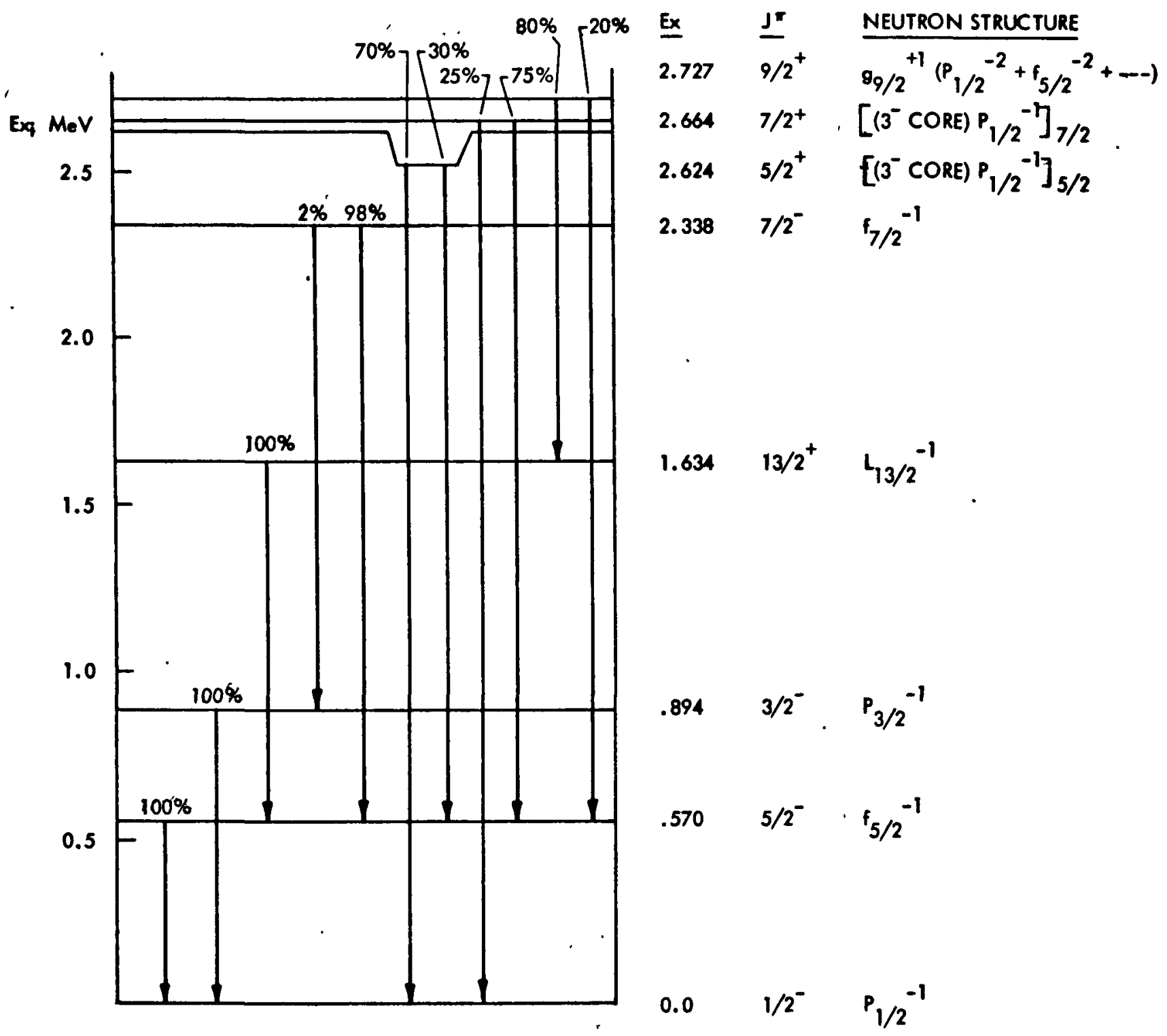

Figure 2-3b. Level Scheme of $\mathrm{Pb}^{207}$ 


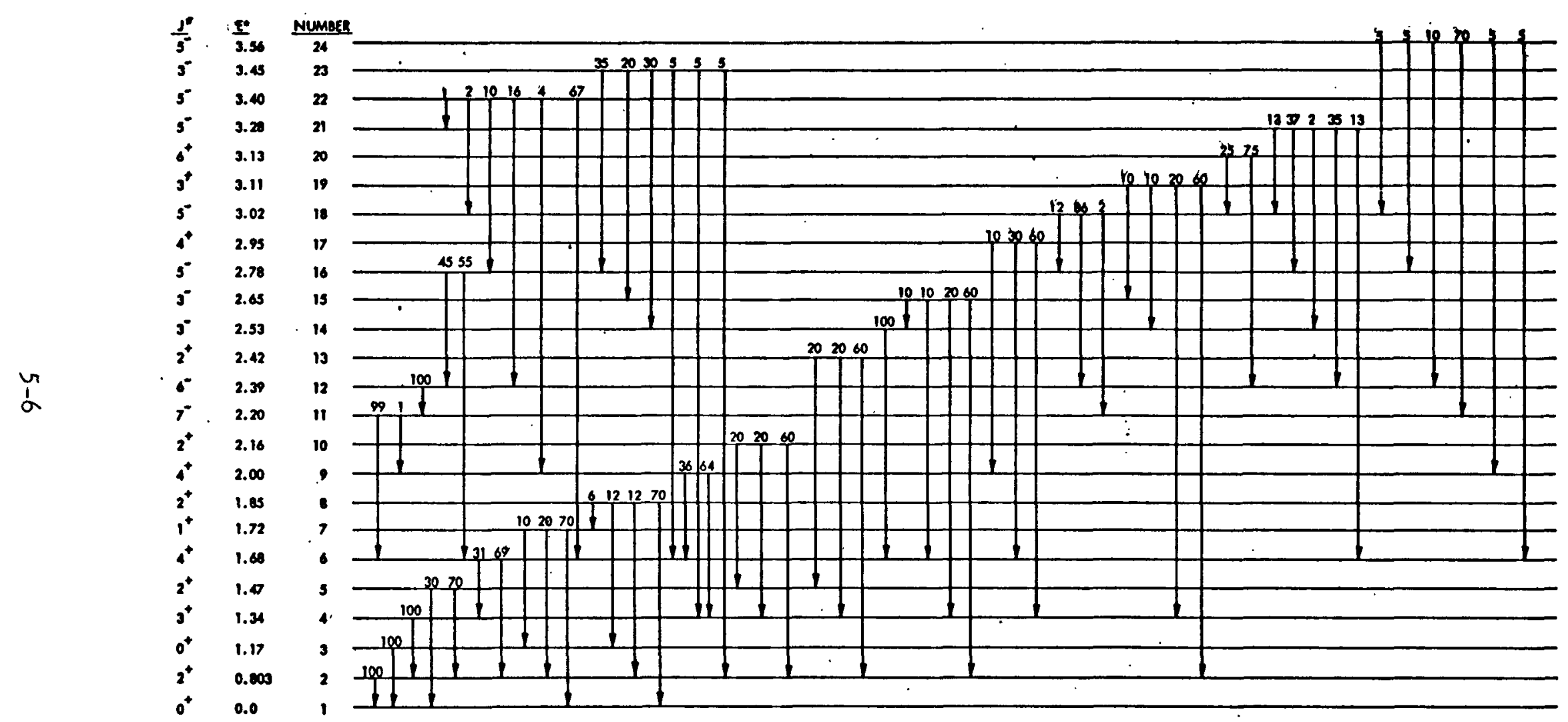

Figure 2-3c. $\mathrm{Pb}^{206}$ Branching Ratios 


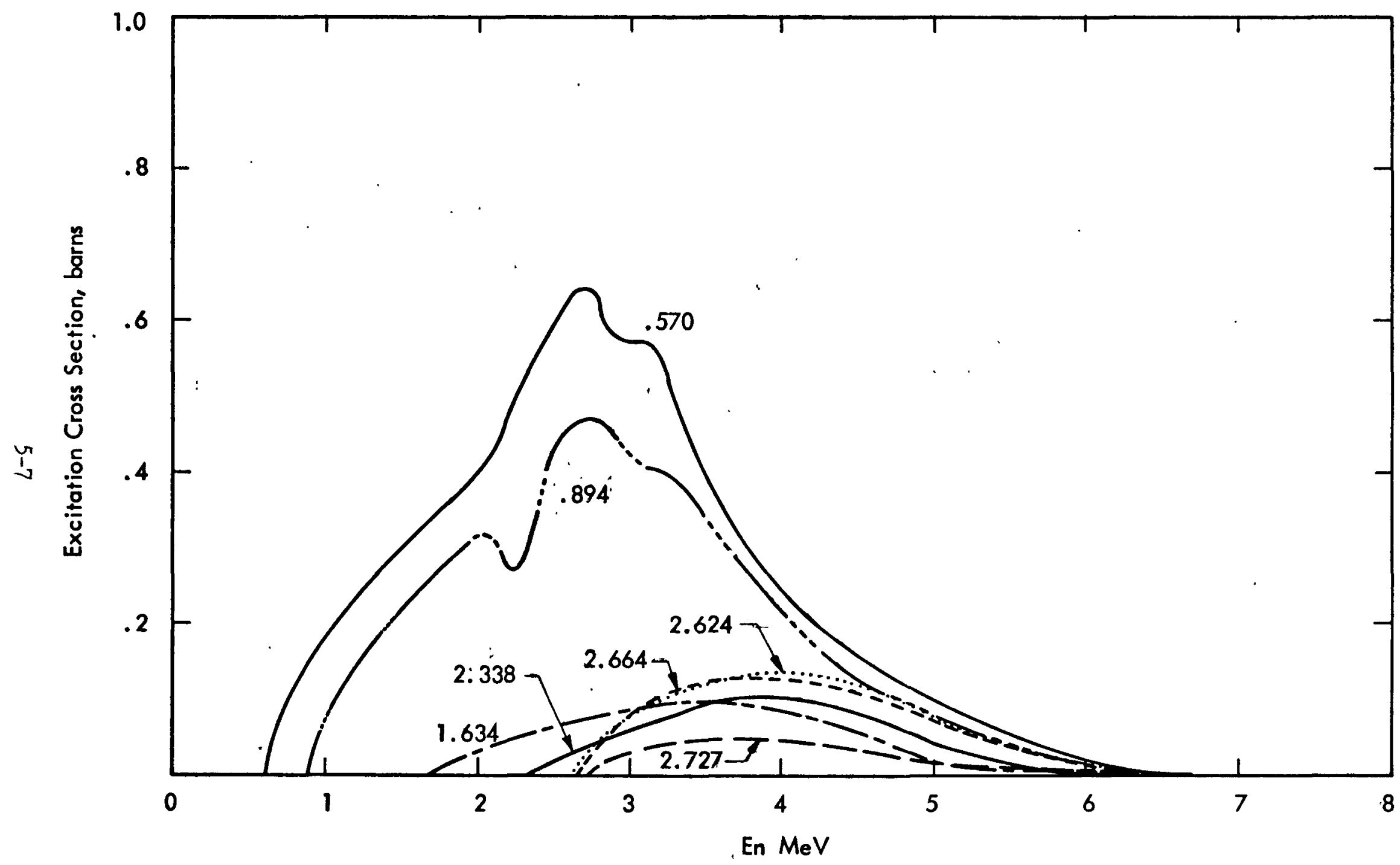

Figure 2-4. $\mathrm{Pb}^{207}\left(n, n^{\prime}\right)$ Input to CASCADE 


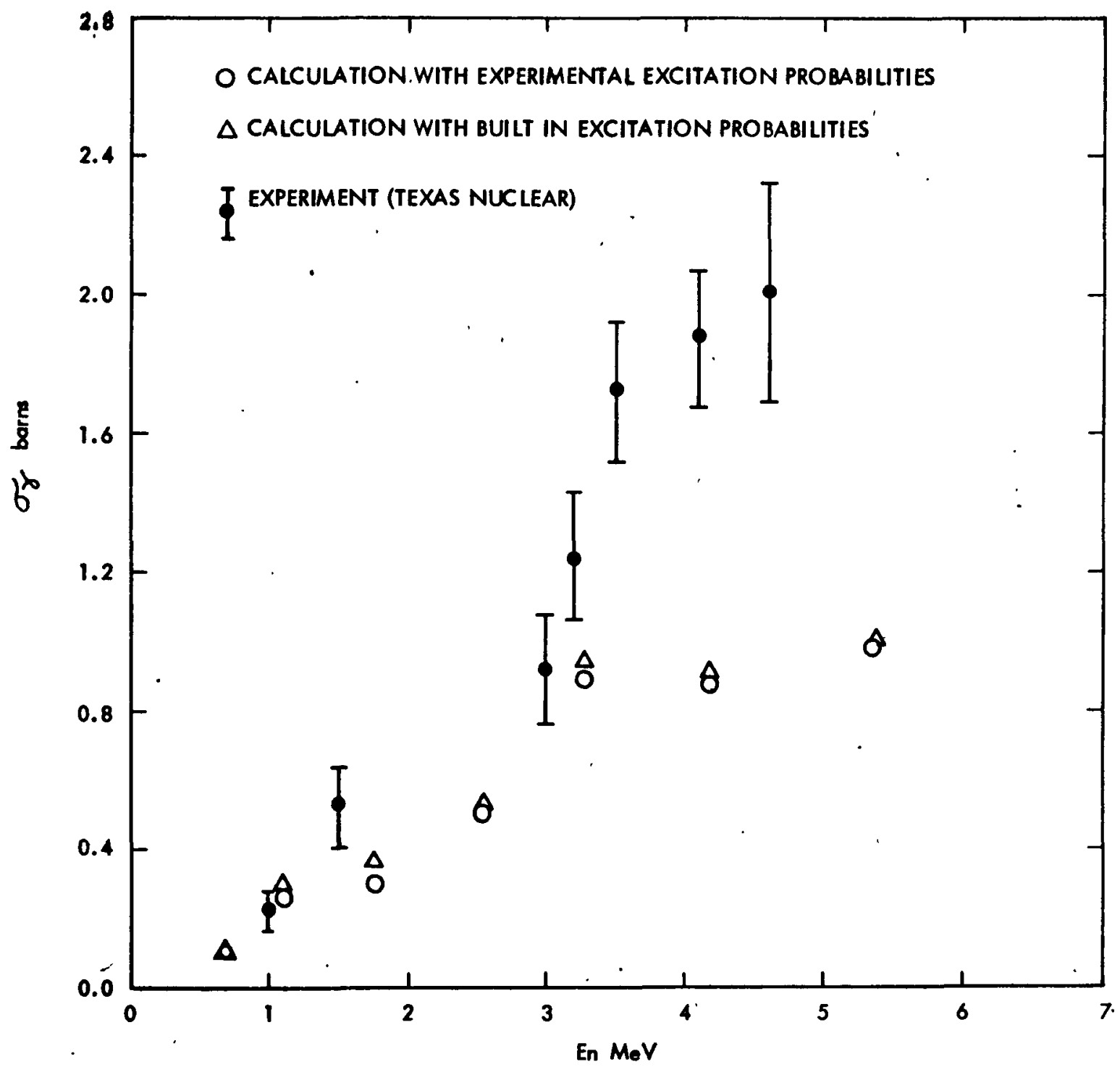

Figure 2-5a. $\mathrm{Pb}^{207}$ Inelastic .57 $\gamma$ Ray 


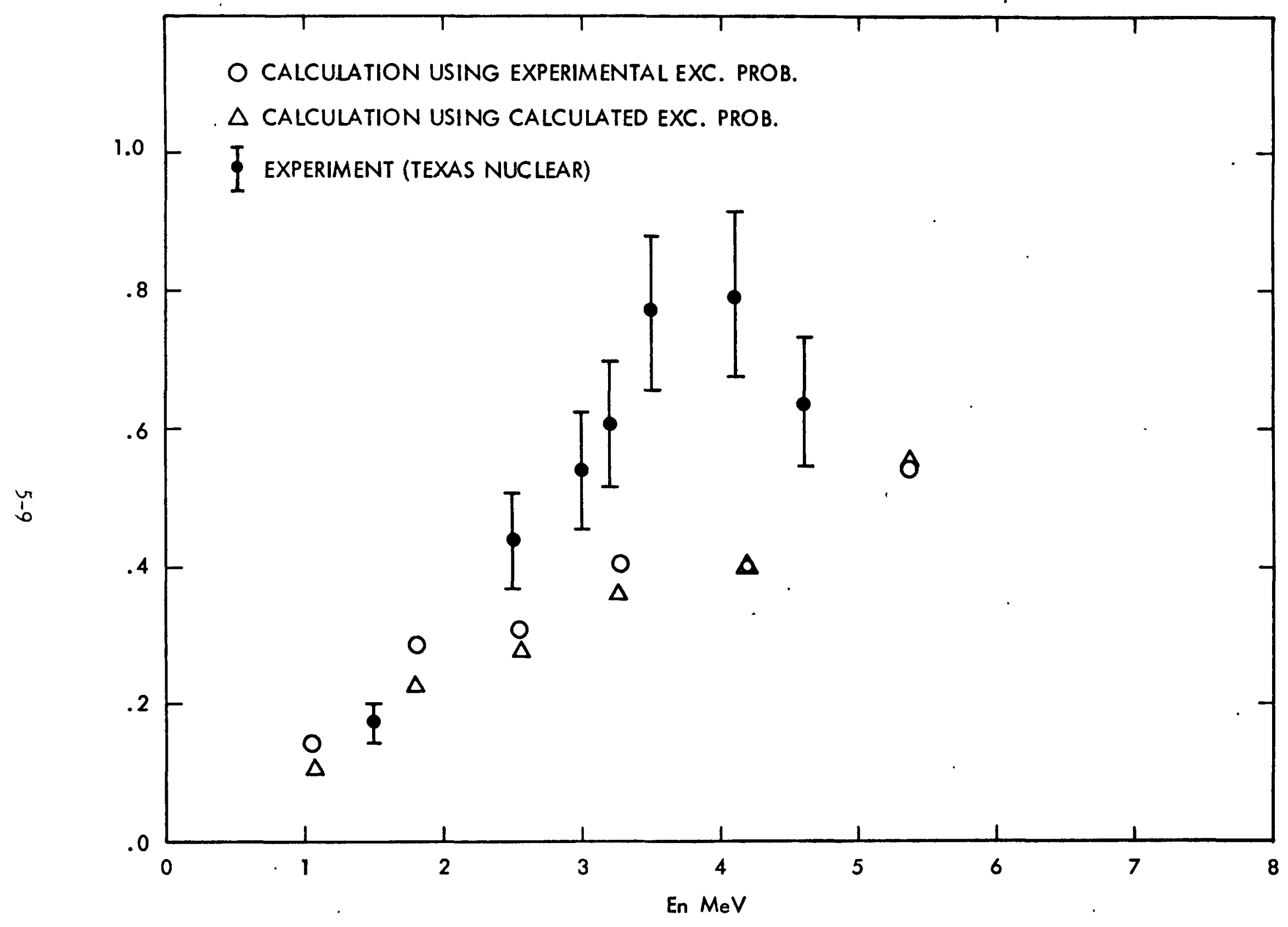

Figure 2-5b. $\mathrm{Pb}^{207}$ Inelastic $.894 \gamma$ Ray 


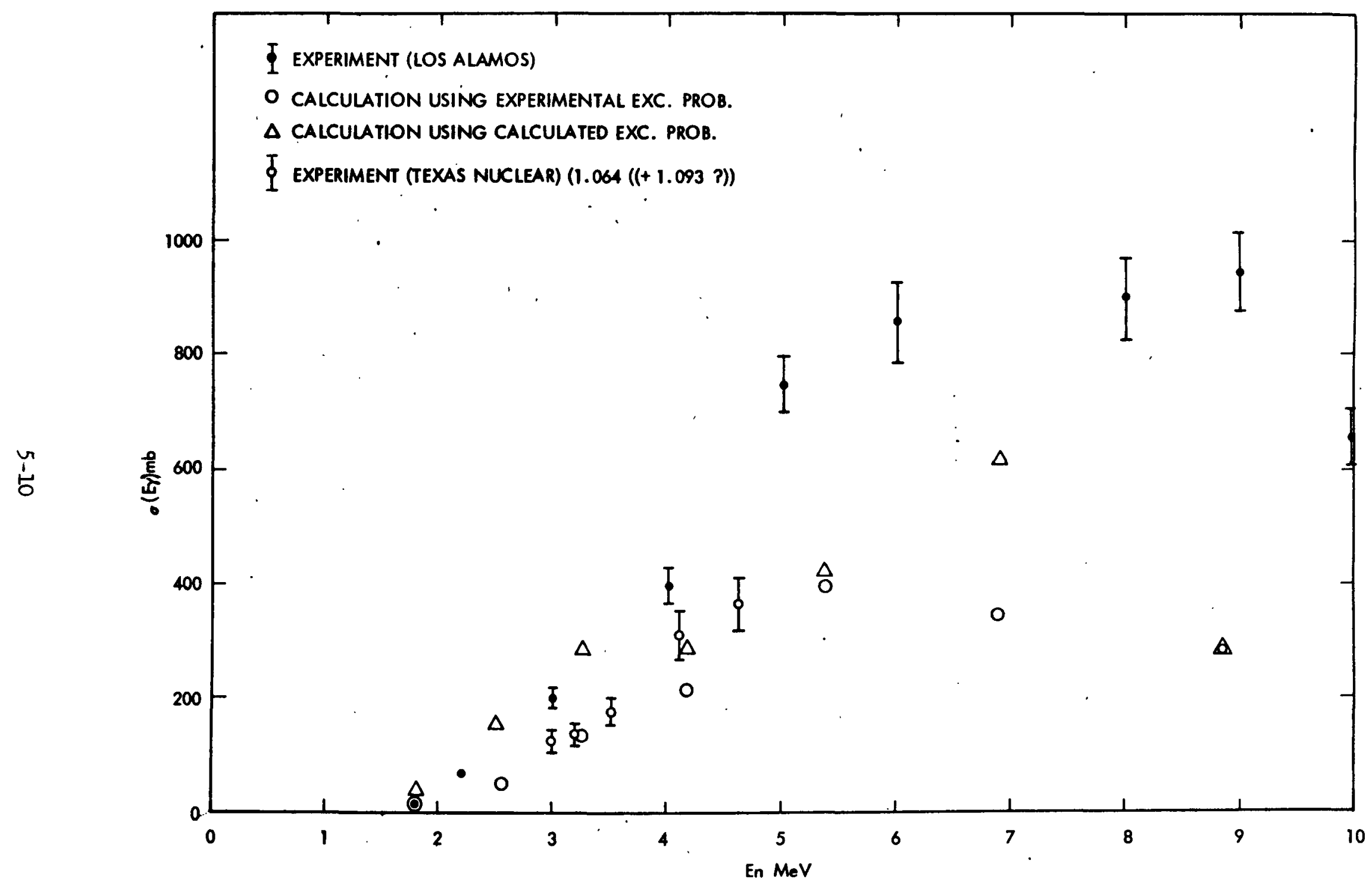

Figure 2-5c. Pb 207 Production of $1.064 \mathrm{MeV}$ - Ray - GAMB405 vs Exp. (Los Alamos) \& Tex. Nuc. 


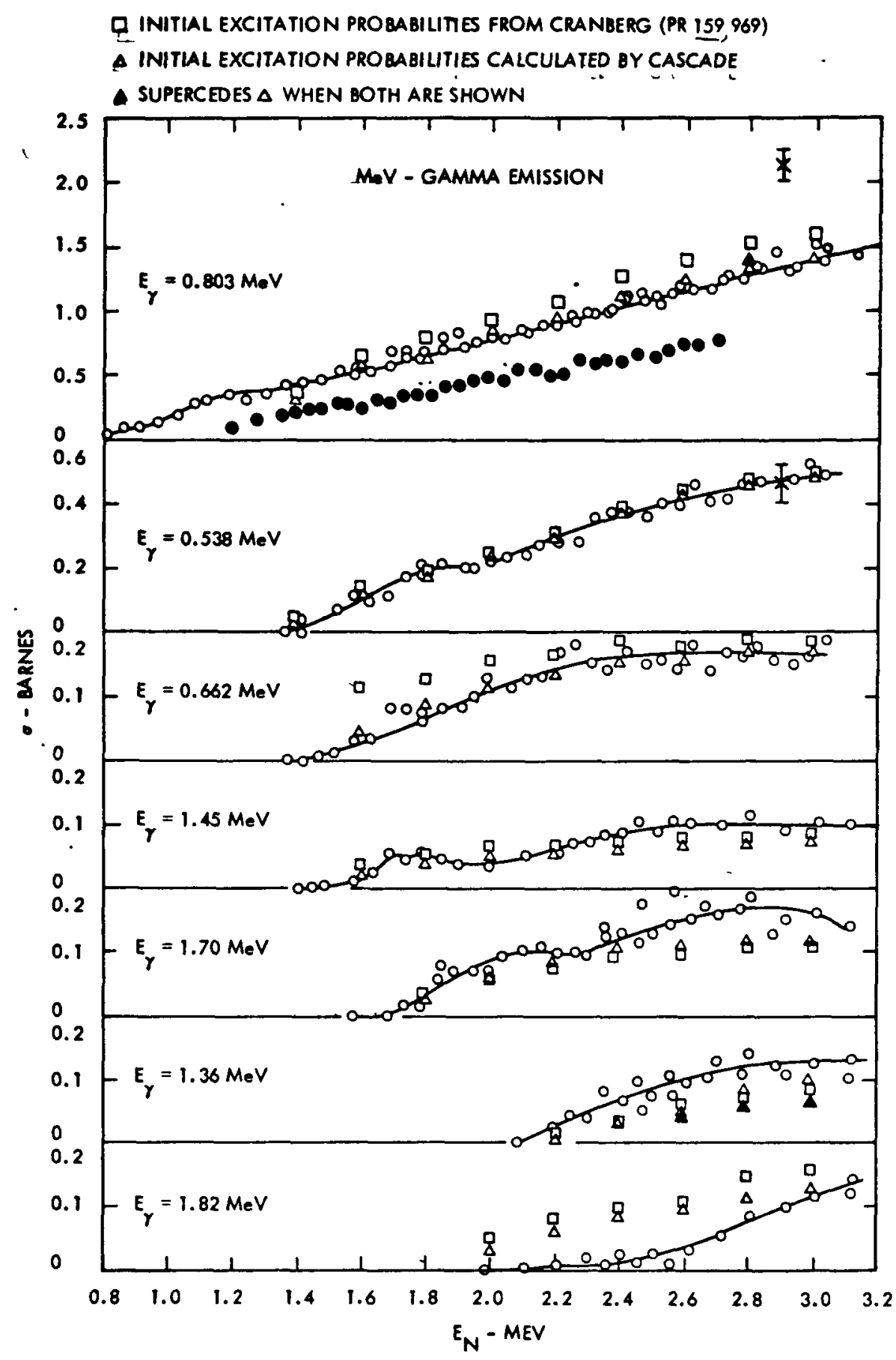

Figure 2 6. CASCADE Results + Cranberg Inelastic C.S. 


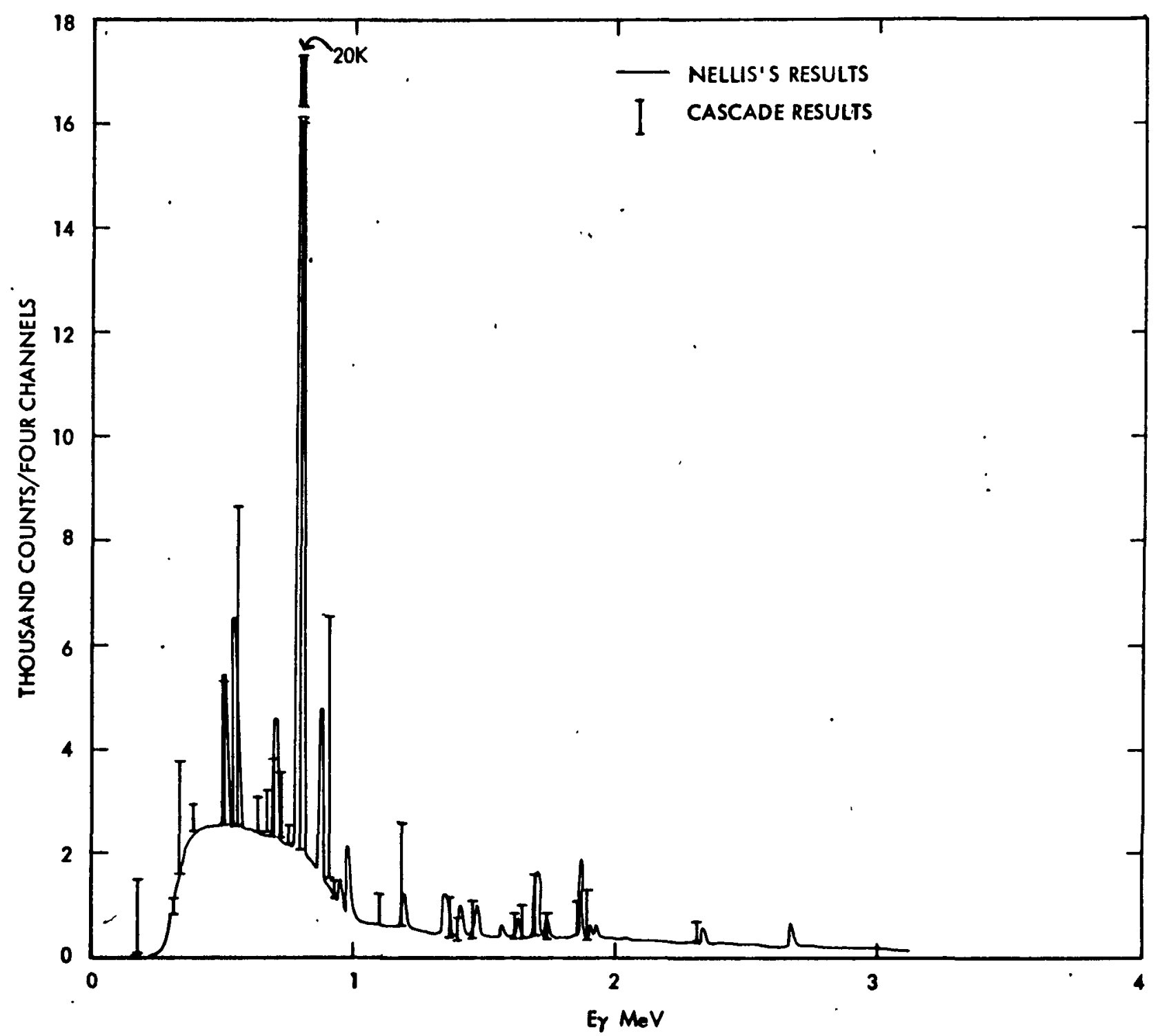

Figure 2-7. Gamma Spectrum from Inelastic Neutron Scattering on ${ }^{206} \mathrm{~Pb}$ 


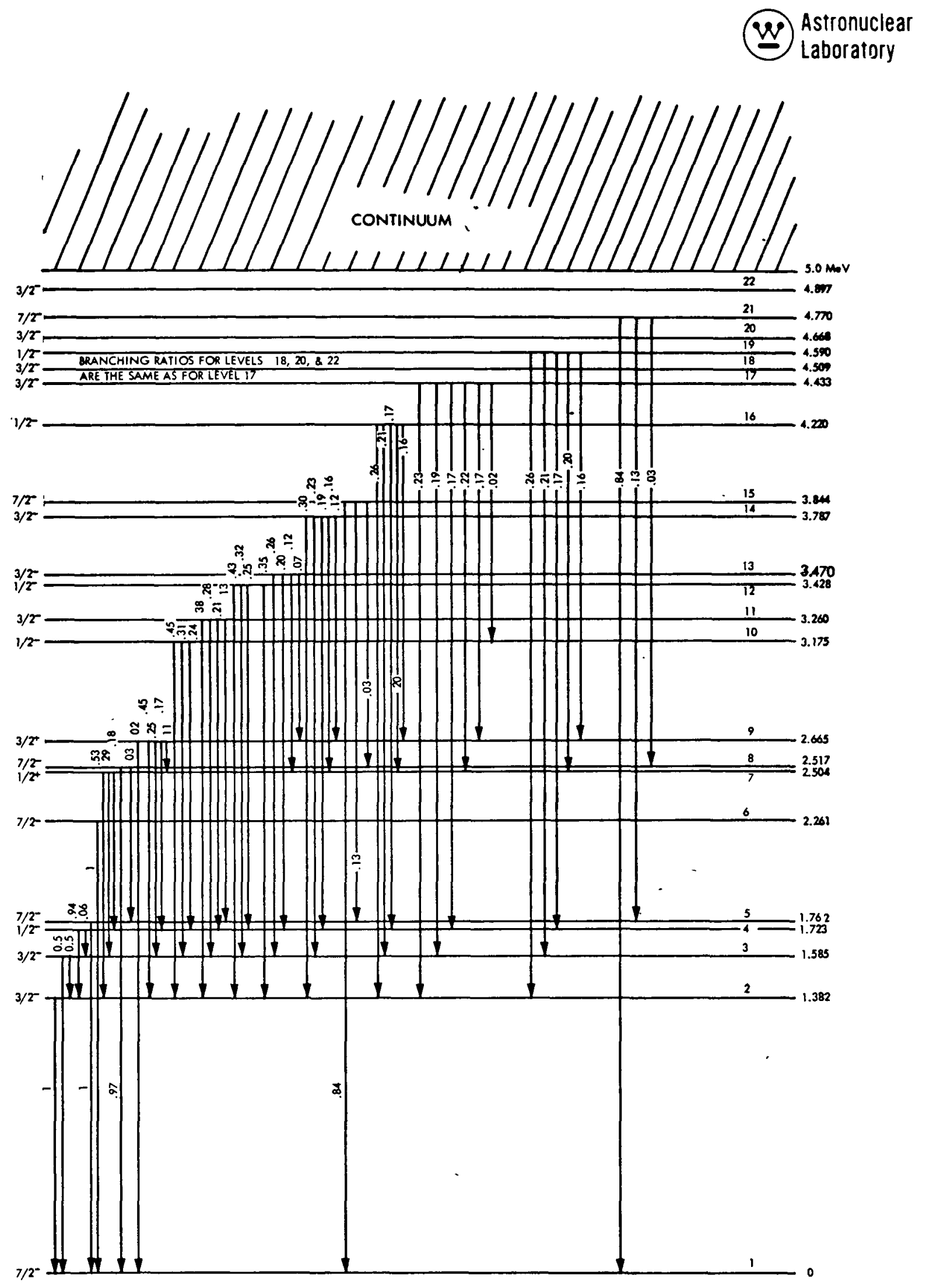

Figure 3-1. Nuclear Levels and Branching Ratios for ${ }^{49} \mathrm{Ti}$ 


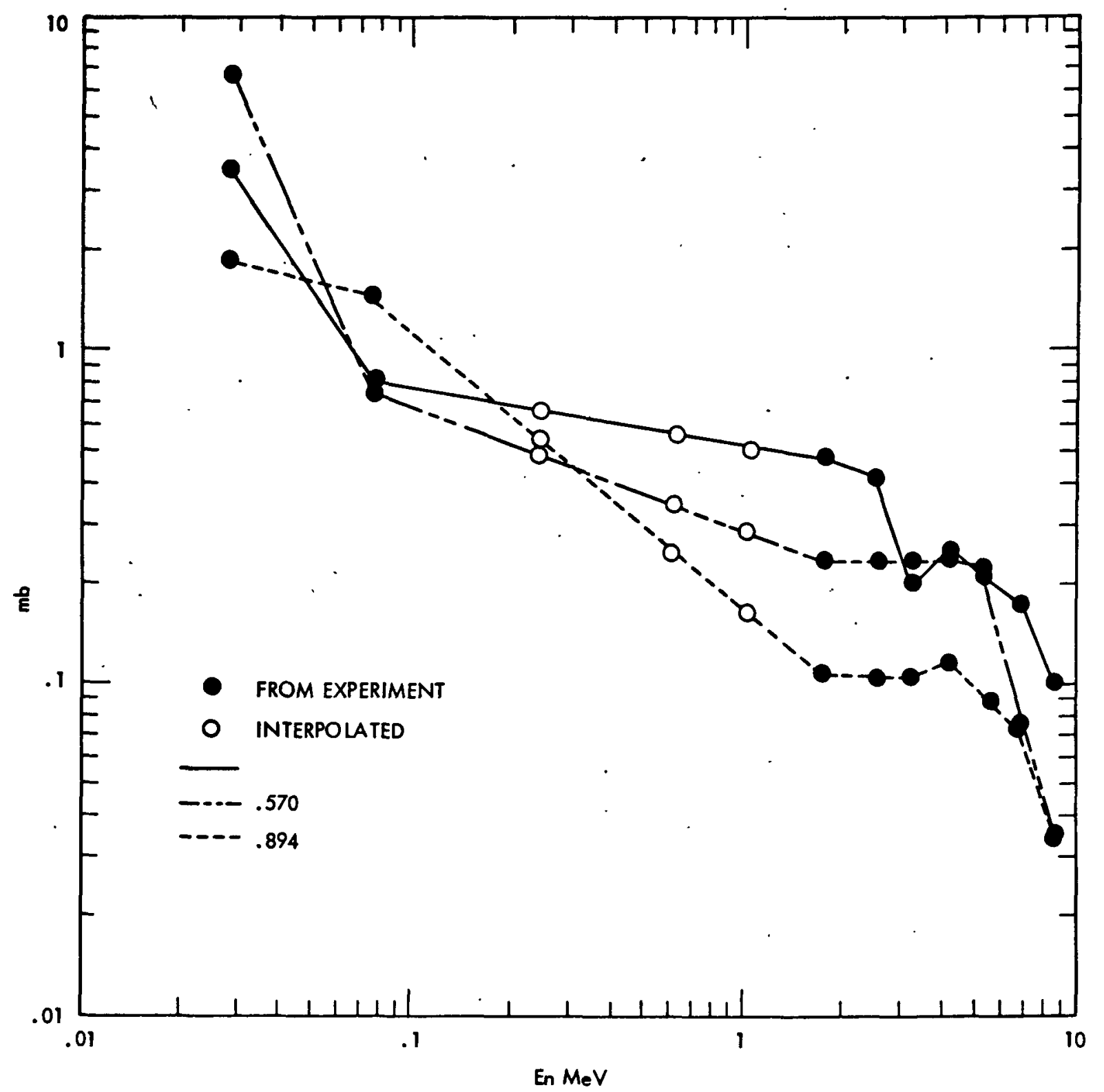

Figure 3-2a. Direct Excitation of Low-Lying Levels in $\mathrm{Pb}^{206}$ Neutron Capture 
W Astronuclear

Laboratory

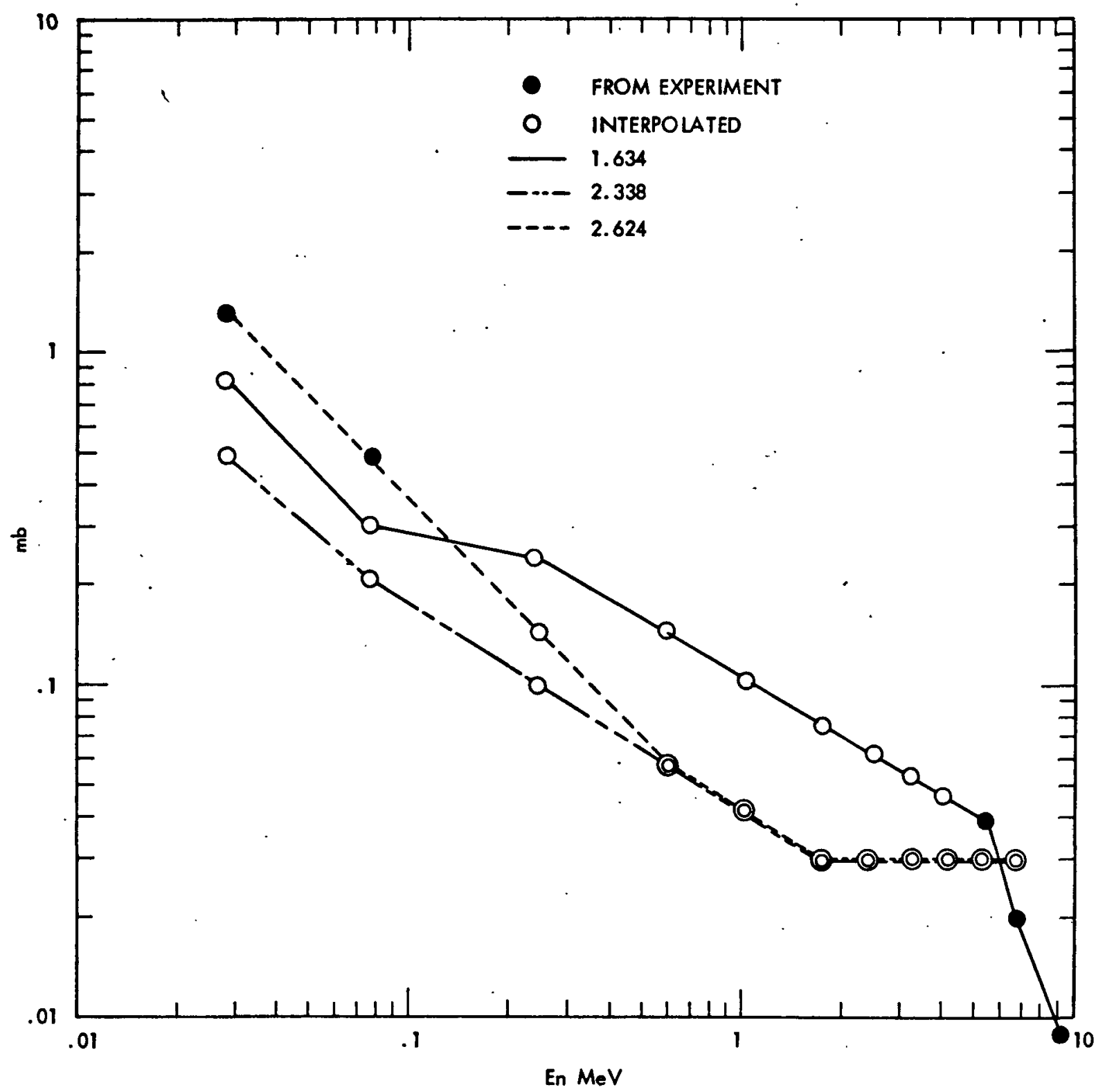

Figure 3-2b. Cross Sections for Excitation of ${ }^{206} \mathrm{~Pb}$ 


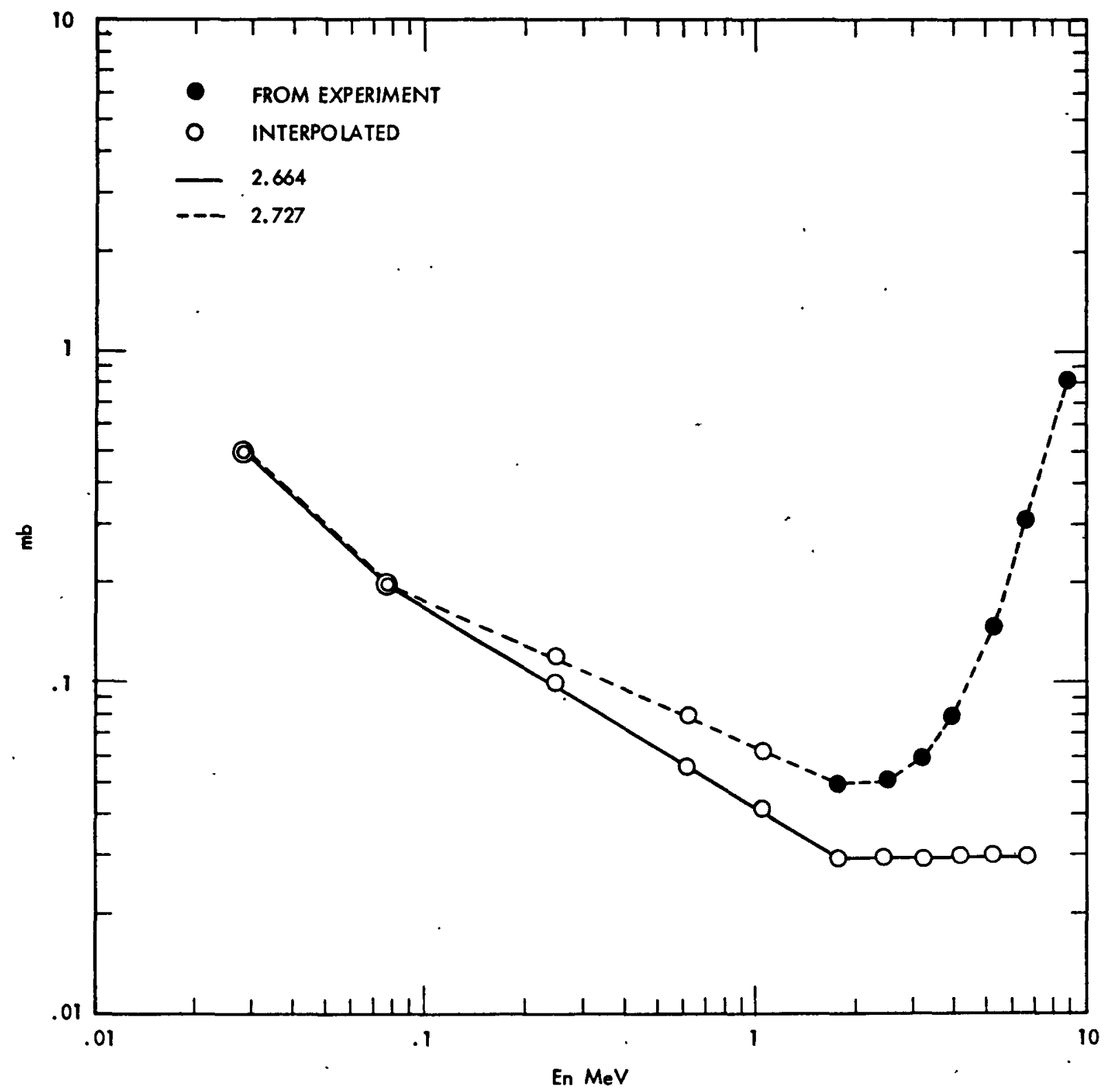

Figure 3-2c. Cross Sections for Excitation of ${ }^{206} \mathrm{~Pb}$ 
(W) Astronuclear

6.0 TABLES

6-1 


\section{TABLE I}

\section{GROUP BOUNDARIES}

\begin{tabular}{cc} 
Group & Boundaries \\
\hline 1 & $10 \mathrm{MeV}$ \\
2 & 7.79 \\
3 & 6.07 \\
4 & 4.72 \\
5 & 3.68 \\
6 & 2.87 \\
7 & 2.23 \\
8 & 1.35 \\
9 & 0.821 \\
10 & 0.388 \\
11 & 0.111 \\
12 & 0.0409 \\
& 0.015 \\
\hline 1 &
\end{tabular}

$13 \gamma$-Groups

\begin{tabular}{cl} 
Group & Boundaries \\
1 & $9.5 \mathrm{MeV}$ \\
2 & 7.5 \\
3 & 7.0 \\
4 & 6.0 \\
5 & 5.0 \\
6 & 4.0 \\
7 & 3.0 \\
8 & 2.6 \\
9 & 2.2 \\
10 & 1.8 \\
11 & 1.35 \\
12 & 0.9 \\
13 & 0.4 \\
\hline 13 & 0
\end{tabular}


(2) Astronuclear Laboratory

TABLE IIa RADIANT ENERGY ( $\underset{27_{\text {Al }}}{(\mathrm{MeV})}$ PER EVENT $\left(n, n^{\prime} \gamma\right)$

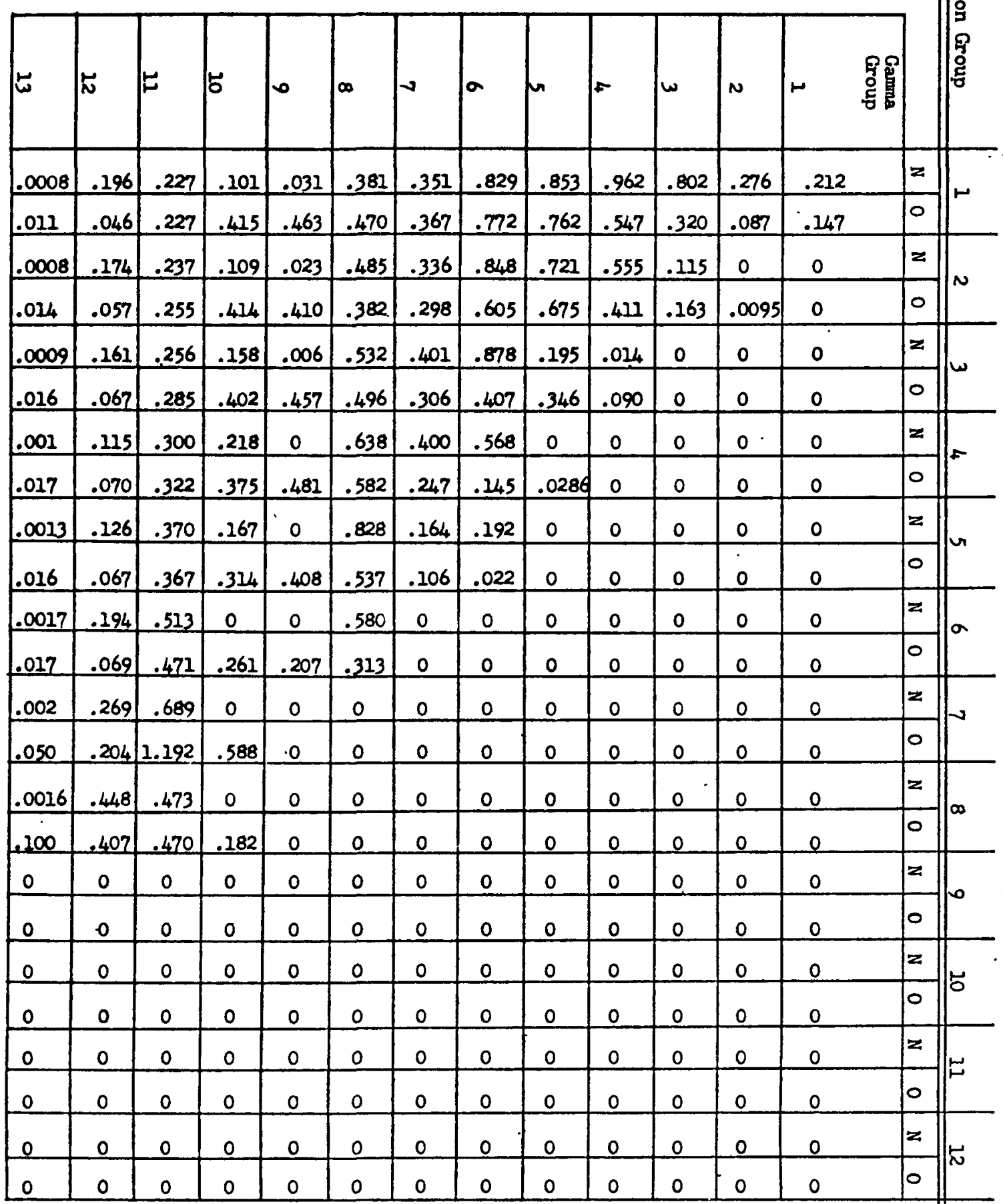

\section{$N$ - Now CASCADE-CASING Results \\ O - POPOP4 Results}


TABLE IIb

RADIANT ENERGY (MeV) PER EVENT ${ }^{56} \mathrm{Fe}$ $\left(n, n^{\prime} \gamma\right)$

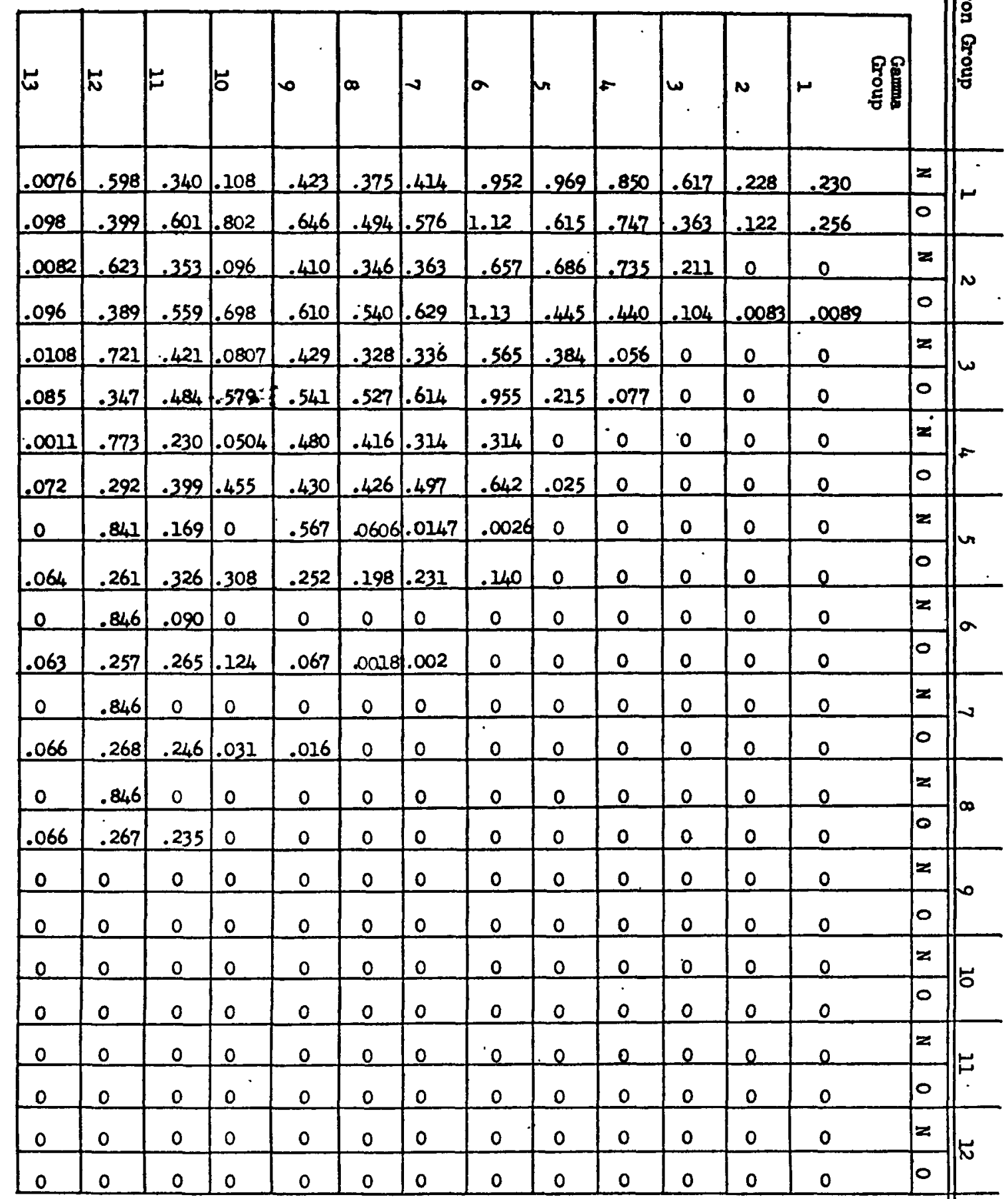

- N - New CASCADE-CASING Results

O - POPOP4 Results 
TABLE IIC

RADIANT ENERGY (MeV) PER EVENT $48_{\text {Ti }}$ *

$\left(n, n^{\prime} \gamma\right)$

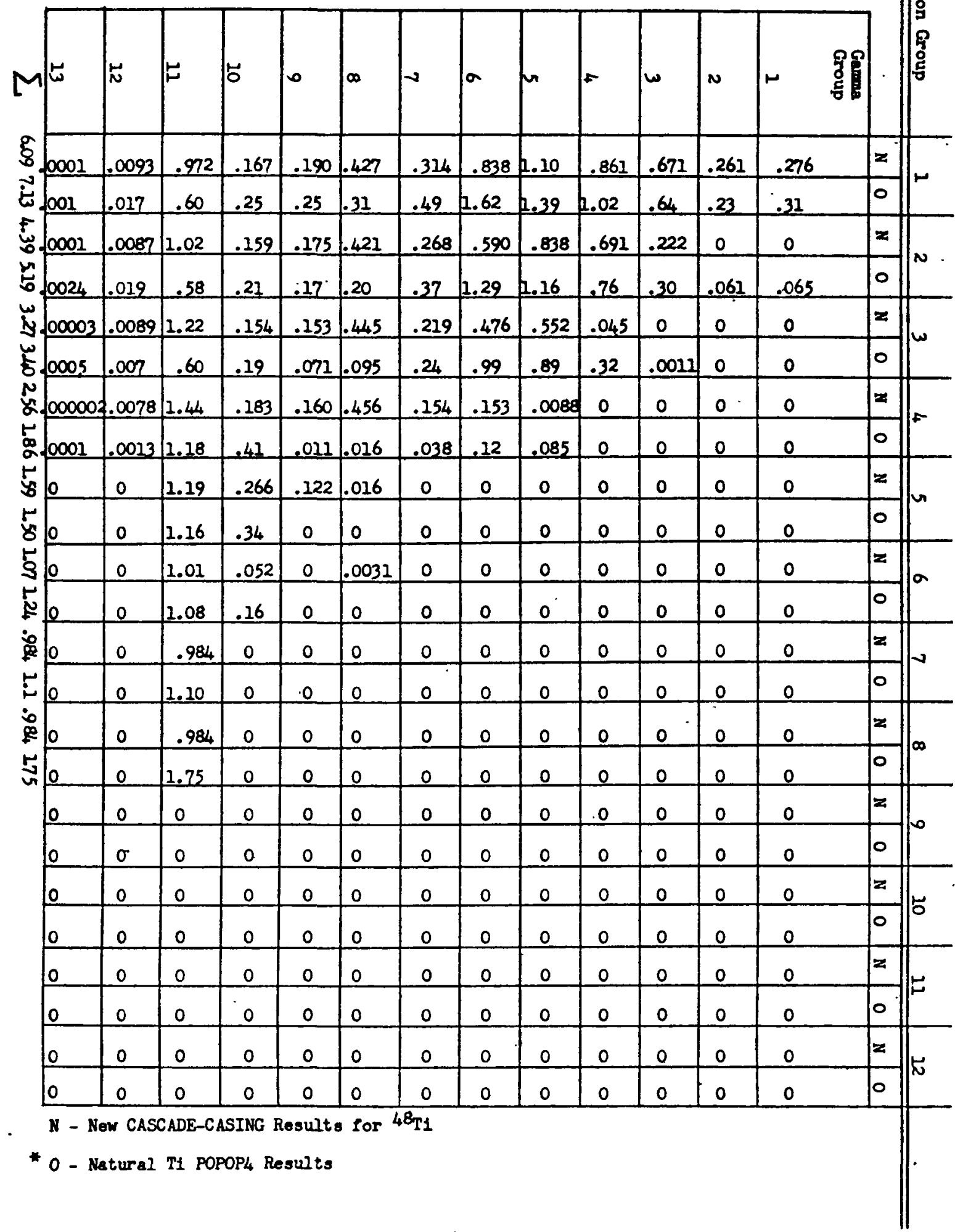


TABLE IId

RADIANT ENERGY (MeV) PER EVENT INELASTIC SCATTERING NAT. PB.

\begin{tabular}{|c|c|c|c|c|c|c|c|c|c|c|c|c|c|}
\hline$\omega$ & $\underline{N}$ & $\boldsymbol{\omega}$ & 하 & $\infty$ & $\infty$ & $\checkmark$ & a & r & $F$ & $\omega$ & $N$ & 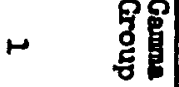 & \\
\hline 0.014 & 0.454 & 0.169 & 0.263 & 0.284 & 0.320 & 0.831 & 0.957 & 1.237 & 1.097 & 0.837 & 0.308 & 0.421 & $z$ \\
\hline 0.025 & b.192 & 0.151 & 0.131 & 0.095 & 0.126 & 0.329 & 0.472 & 0.574 & 0.290 & 0.610 & 0 & 0 & 0 \\
\hline 0.014 & 0.444 & 0.124 & 0.171 & 0.167 & 0.188 & 0.696 & 0.650 & 0.858 & 1.160 & 0.776 & 0 & 0 & $=$ \\
\hline 0.004 & 0.253 & 0.180 & 0.219 & 0.191 & 0.263 & 0.705 & 0.676 & 0.931 & 0.270 & 0.338 & 0 & 0 & 0 \\
\hline 0.020 & b.553 & 0.100 & 0.133 & 0.112 & 0.108 & 0.919 & 0.399 & 1.140 & 0.258 & 0 & 0 & 0 & $z$ \\
\hline 0.005 & 0.307 & 0.212 & 0.261 & 0.200 & 0.272 & 0.886 & 0.626 & 0.594 & 0.021 & 0.004 & 0 & 0 & 0 \\
\hline 0.024 & 0.662 & 0.090 & 0.112 & 0.086 & 0.062 & 1.508 & 0.219 & 0.011 & 0 & 0 & 0 & 0 & $z$ \\
\hline 0.004 & b.357 & 0.232 & 0.236 & 0.151 & 0.211 & 0.824 & 0.311 & 0.107 & 0 & 0 & 0 & 0 & 0 \\
\hline 0.009 & 0.421 & 0.083 & 0.093 & 0.075 & 0.023 & 1.449 & 0.018 & 0 & 0 & 0 & 0 & 0 & $z$ \\
\hline 0.002 & 0.426 & 0.239 & 0.176 & 0.099 & 0.136 & 0.588 & 0.024 & 0 & 0 & 0 & 0 & 0 & 0 \\
\hline 0.005 & 0.382 & 0.071 & 0.061 & 0.024 & 0 & 1.378 & 0 & 0 & 0 & 0 & 0 & 0 & $z$ \\
\hline 0 & 0.589 & 0.259 & 0.116 & 0.066 & 0.034 & 0.148 & 0 & 0 & 0 & 0 & 0 & 0 & 0 \\
\hline 0.001 & 0.348 & 0.079 & 0.021 & 0 & 0 & 0 & 0 & 0 & 0 & 0 & 0 & 0 & $z$ \\
\hline 0 & 0.605 & 0.223 & 0.021 & 0.009 & 0 & 0 & $\underline{0}$ & 0 & 0 & 0 & 0 & 0 & 0 \\
\hline 0 & b.352 & 0 & 0 & 0 & 0 & 0 & 오 & $e$ & 0 & 0 & 0 & 0 & $z$ \\
\hline 0 & 0.345 & 0.116 & 0 & 0 & 0 & 0 & e & 0 & 0 & 0 & 0 & 0 & 0 \\
\hline 0 & 0.328 & 0 & 0 & 0 & 0 & 0 & 0 & 0 & 0 & 0 & 0 & 0 & $z$ \\
\hline 0 & 0 & 0 & 0 & 0 & 0 & 0 & 0 & 0 & 0 & 0 & 0 & 0 & 10 \\
\hline 0 & 0 & 0 & 0 & 0 & 0 & 0 & 0 & 0 & 0 & 0 & 0 & 0 & $z$ \\
\hline 0 & 0 & 0 & 0 & 0 & 0 & 0 & 0 & 0 & 0 & 0 & 0 & 0 & 0 \\
\hline 0 & 0 & 0 & 0 & 0 & 0 & 0 & 0 & 0 & 0 & 0 & 0 & 0 & $z$ \\
\hline 0 & 0. & 0 & 0 & 0 & 0 & 0 & 0 & 0 & 0 & 0 & 0 & 0 & 0 \\
\hline 0 & 0 & 0 & 0 & 0 & 0 & 0 & 0 & 0 & 0 & 0 & 0 & 0 & $z$ \\
\hline 0 & 0 & 0 & 0 & 0 & 0 & 0 & 0 & 0 & 0 & 0 & 0 & 0 & 0 \\
\hline $0-$ & OPOP4 & $=r$ & & sults & & & & & & & & & \\
\hline
\end{tabular}


W Astronuclear

TABLE IIIa

RADIANT ENERGY (Mgly) PER EVENT $\left(n, n^{\prime} \gamma\right)$

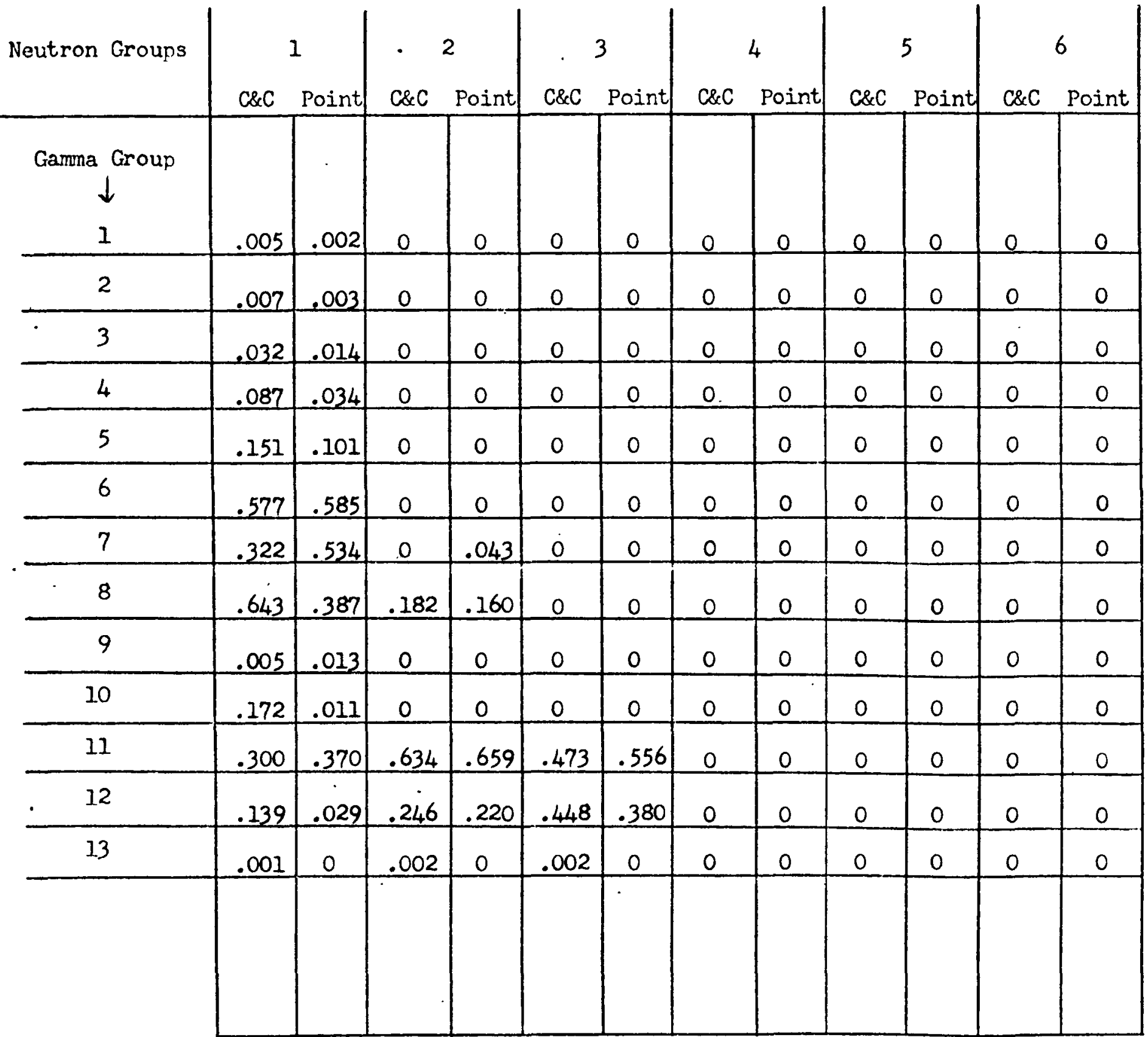


TABLE IITb

RADIANT ENERGY (MegV) PER EVENT $\left(n, n^{\prime} \gamma\right)$

\begin{tabular}{|c|c|c|c|c|c|c|c|c|c|c|c|c|}
\hline \multirow[t]{2}{*}{ Neutron Croups } & \multicolumn{2}{|c|}{1} & \multicolumn{2}{|c|}{2} & \multicolumn{2}{|c|}{3} & \multicolumn{2}{|c|}{4} & \multicolumn{2}{|c|}{5} & \multicolumn{2}{|c|}{6} \\
\hline & $\mathrm{CSSC}$ & Point & $\mathrm{CE} C$ & Point & $\mathrm{C \& C}$ & Point. & $\mathrm{CsC}$ & Point & $C \& C$ & Point & $\mathrm{C \& C}$ & Point \\
\hline $\begin{array}{c}\text { Gamma Group } \\
\downarrow\end{array}$ & & & & & & & & & & & & \\
\hline 1 & .0054 & 0 & 0 & 0 & 0 & 0 & 0 & 0 & 0 & 0 & 0 & 0 \\
\hline 2 & .0054 & 0 & 0 & 0 & 0 & 0 & 0 & 0 & 0 & 0 & 0 & 0 \\
\hline 3 & .0350 & 0 & 0 & 0 & 0 & 0 & 0 & 0 & 0 & 0 & 0 & 0 \\
\hline 4 & .1056 & 0 & 0 & 0 & 0 & 0 & 0. & 0 & 0 & 0 & 0 & 0 \\
\hline 5 & .1866 & 0 & 0 & 0 & 0 & 0 & 0 & 0 & 0 & 0 & 0 & 0 \\
\hline 6 & .3215 & 1.628 & 0 & 0 & 0 & 0 & 0 & 0 & 0 & 0 & 0 & 0 \\
\hline 7 & .2266 & 0 & 0 & .0887 & 0 & 0 & 0 & 0 & 0 & 0 & 0 & 0 \\
\hline 8 & .2668 & 0 & 0 & 0 & 0 & 0 & 0 & 0 & 0 & 0 & 0 & 0 \\
\hline 9 & .4883 & 1.007 & 0 & 0 & 0 & 0 & 0 & 0 & 0 & 0 & 0 & 0 \\
\hline 10 & .0470 & 0 & 0 & 0 & 0 & 0 & 0 & 0 & 0 & 0 & 0 & 0 \\
\hline 11 & .2724 & .620 & .0238 & 0 & 0 & 0 & 0 & 0 & 0 & 0 & 0 & 0 \\
\hline 12 & .7640 & .845 & .8460 & .819 & .846 & .845 & 0 & 0 & 0 & 0 & 0 & 0 \\
\hline 13 & .0040 & 0 & .0 & 0 & 0 & 0 & 0 & 0 & 0 & 0 & 0 & 0 \\
\hline$\sum$ & 2.728 & 4.100 & .8698 & .9077 & .846 & .845 & 0 & 0 & 0 & 0 & 0 & 0 \\
\hline
\end{tabular}




\section{(W) Astronuclear}

TABLE IIIc

RADIANT ENERGY (MeV) $\underset{4 \mathrm{Ti}^{*}}{\text { PER }}$ EVENT $\left(n, n^{\prime} \gamma\right)$

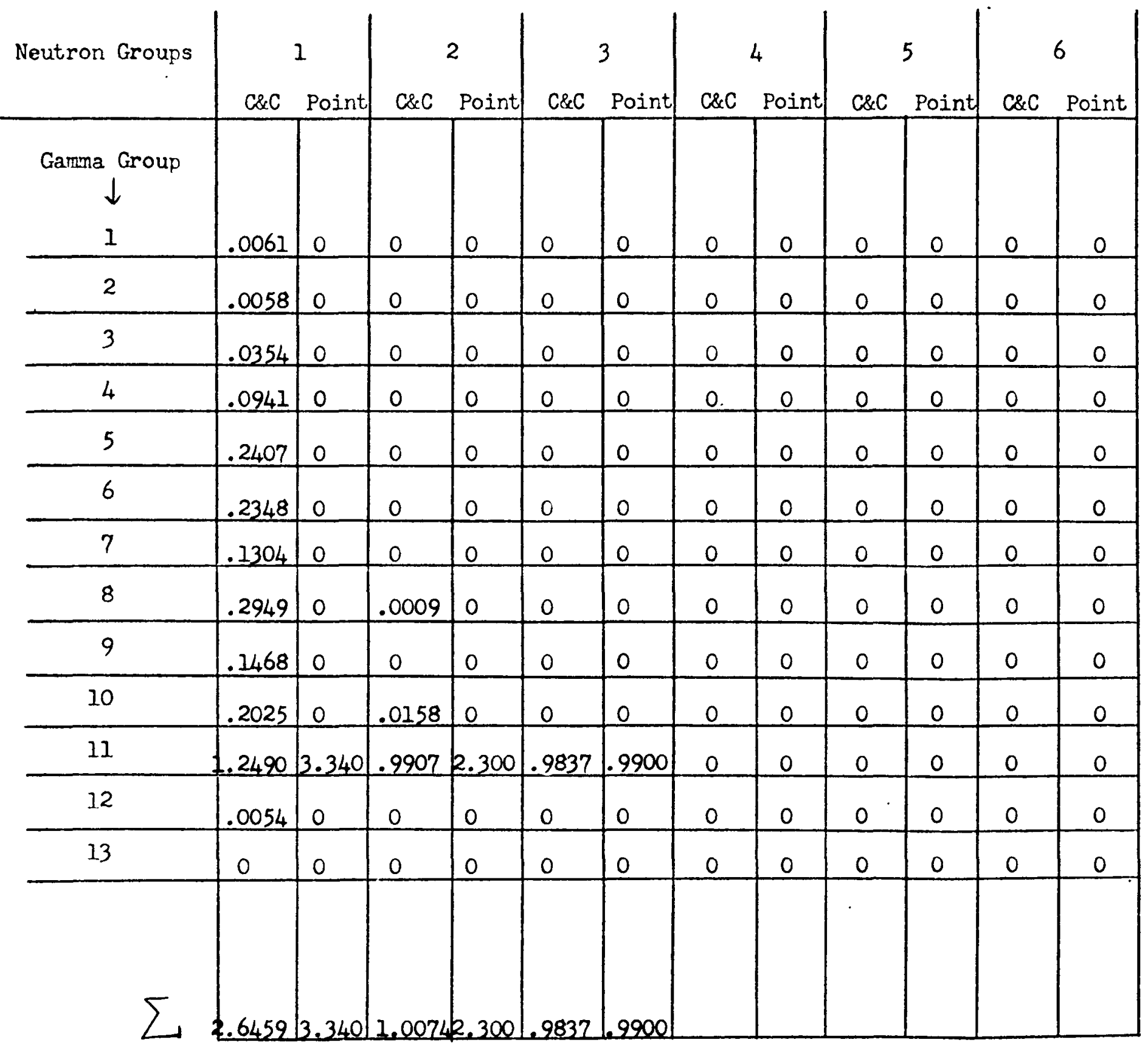

* Natural Ti in POINT Library 
TABIE IIId

RADIANT ENERGY (MeV) PER EVENT $\left(n, n^{\prime} \gamma\right)$

NAT. PB.

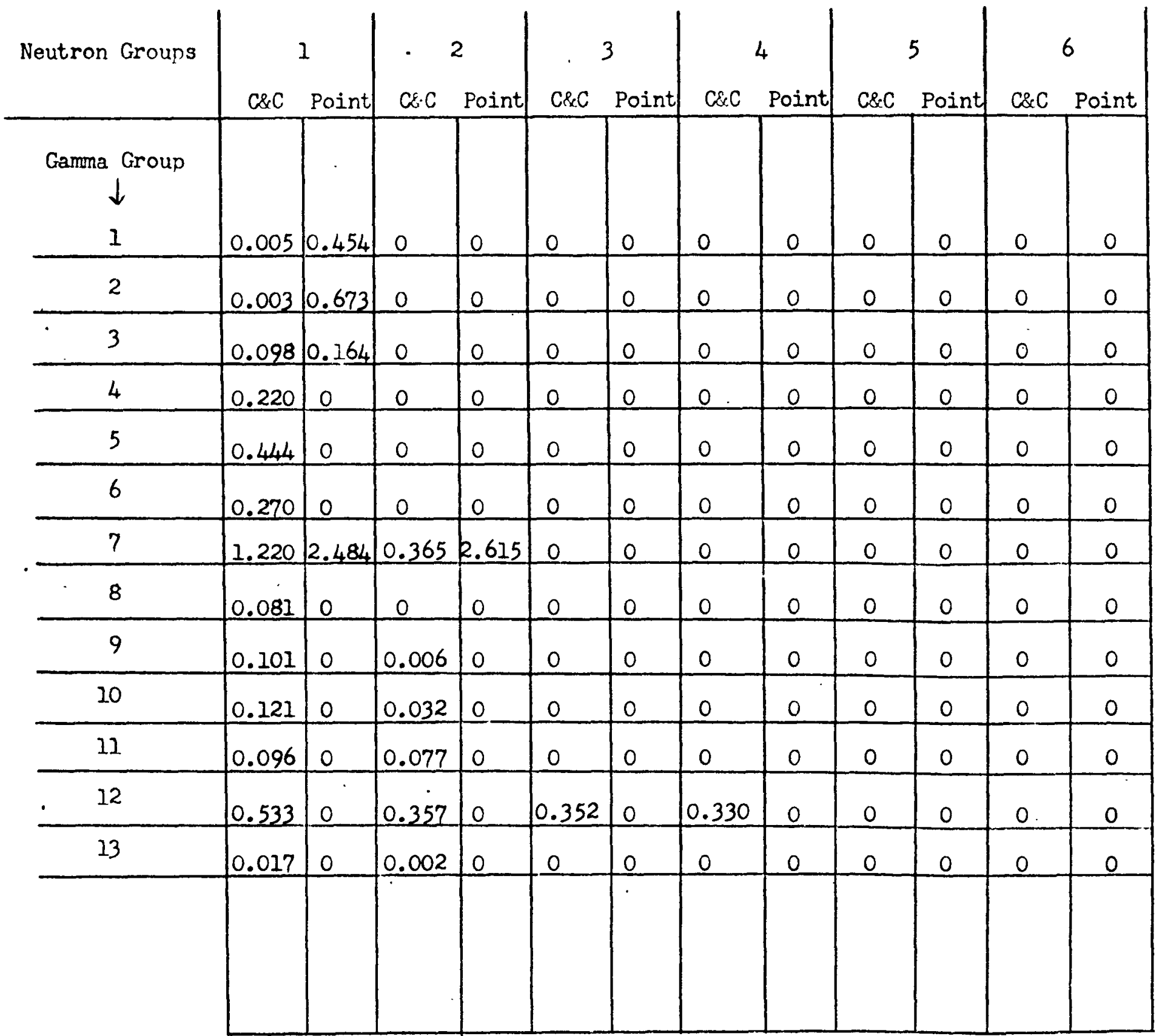




\section{TABLE IV}

OPTICAL PARAMETERS USED IN HELENE

Set 1 Moore $1 \mathrm{MeV}$ Optical Parameters - Gaussian imaginary well, Woods-Saxon real well.

$$
\begin{aligned}
& \text { real depth } \quad=52 \mathrm{MeV} \\
& \text { " radius }=1.175 \mathrm{fm} \\
& \text { "diffusivity }=.55 \mathrm{fm} \\
& \begin{array}{rll}
\text { imaginary depth } & =2 \mathrm{MeV} \\
" & \text { radius } & =1.175 \mathrm{fm} \\
" & \text { diffusivity } & =1.0 \mathrm{fm}
\end{array}
\end{aligned}
$$

Set 2 Moore $2.5 \mathrm{MeV}$ Parameters. Same well types as above.

$$
\begin{aligned}
& \text { real depth } \quad=46 \mathrm{MeV} \\
& " \text { radius }=1.25 \mathrm{fm} \\
& \text { " diffusivity }=.65 \mathrm{fm}
\end{aligned}
$$

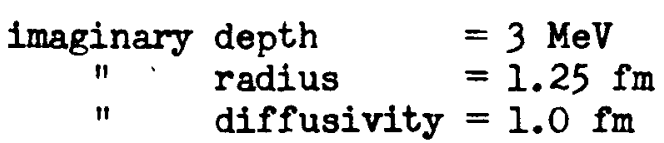

Set 3 Greenlees $5 \mathrm{MeV}$ Parameters - Real and imaginary wells - Woods-Saxon (Imaginary well is $W-S$ derivative!)

$$
\begin{aligned}
\text { real depth } & =49.7 \mathrm{MeV} \\
\text { " radius } & =1.17 \mathrm{fm} \\
\text { " diffusivity } & =.75 \mathrm{fm} \\
\text { imaginary depth } & =9.26 \mathrm{MeV} \\
" \text { " radius } & =1.26 \mathrm{fm} \\
\text { " diffusivity } & =.58 \mathrm{fm}
\end{aligned}
$$




\section{TABLE V}

RADIANT ENERGY (MeV) EMITTED PER THERMAL NEUTRON CAPTURE IN Ti, I.E. THE $Q(n, \gamma)$ MATRIX

\begin{tabular}{|c|c|c|c|}
\hline X-Ray Group & C\&C Results* & Fody's Results & Point \\
\hline 1 & 0.0006 & 0.0575 & 0.0000 \\
\hline 2 & 0.0045 & 0.0786 & 0.0000 \\
\hline 3 & 5.3500 & 5.4600 & 3.6000 \\
\hline 4 & 0.1640 & 0.0700 & 0.2380 \\
\hline 5 & 0.5850 & 0.5390 & 0.7170 \\
\hline 6 & 0.1280 & 0.2830 & 0.8350 \\
\hline 7 & 0.0840 & 0.0412 & 0.2670 \\
\hline 8 & 0.0795 & 0.0000 & 0.1760 \\
\hline 9 & 0.1000 & 0.0140 & 0.2540 \\
\hline 10 & 1.5000 & 1.5700 & 1.3100 \\
\hline 11 & 0.0263 & 0.0400 & 0.8490 \\
\hline 12 & 0.0034 & 0.0000 & 0.1270 \\
\hline 13 & 0.1270 & 0.1135 & 0.0771 \\
\hline
\end{tabular}


TABLE VI

RADIANT ENERGY (MeV) EMITTED PER NEUTRON CAPTURE IN 48 Ti, I.E. THE $Q(n, \gamma)$ MATRIX FOR 12 STANDARD NEUTRON GROUPS AND $17 \gamma$-GROUPS AS LISTED IN THE TABLE

\begin{tabular}{|l|c|c|c|c|c|c|c|c|c|c|c|c|}
\hline $\begin{array}{c}\text { Upper } \\
\gamma \text {-Group }\end{array}$ & 1 & 2 & 3 & 4 & 5 & 6 & 7 & 8 & 9 & 10 & 11 & 12 \\
\hline $17.5 \mathrm{MeV}$ & .02 & 0 & 0 & 0 & 0 & 0 & 0 & 0 & 0 & 0 & 0 & 0 \\
\hline 15.5 & .14 & .08 & 0 & 0 & 0 & 0 & 0 & 0 & 0 & 0 & 0 & 0 \\
\hline 13.5 & .27 & .28 & 0.33 & 0.16 & 0 & 0 & 0 & 0 & 0 & 0 & 0 & 0 \\
\hline 11.5 & 1.11 & .59 & 0.49 & 0.69 & 0.77 & 0.33 & 0.44 & 0 & 0 & 0 & 0 & 0 \\
\hline 9.5 & 2.42 & 2.09 & 1.46 & 0.93 & 1.05 & 1.35 & 1.15 & 1.23 & 0.73 & 0.81 & 0.86 & 0.88 \\
\hline 7.5 & 0.91 & 0.78 & 0.72 & 0.58 & 0.28 & 0.47 & 0.53 & 0.58 & 1.02 & 0.42 & 0.02 & 0.02 \\
\hline 7.0 & 2.20 & 1.93 & 1.78 & 1.71 & 1.20 & 0.89 & 0.96 & 1.12 & 1.29 & 1.41 & 1.61 & 1.61 \\
\hline 6.0 & 2.63 & 2.37 & 2.17 & 2.08 & 2.02 & 1.80 & 1.14 & 1.13 & 1.00 & 1.30 & 1.40 & 1.25 \\
\hline 5.0 & 2.33 & 2.13 & 1.94 & 1.83 & 1.75 & 1.75 & 1.62 & 1.05 & 0.84 & 0.81 & 0.88 & 0.93 \\
\hline 4.0 & 1.91 & 1.80 & 1.69 & 1.59 & 1.51 & 1.48 & 1.45 & 1.44 & 1.21 & 1.04 & 0.88 & 0.84 \\
\hline 3.0 & 0.51 & 0.48 & 0.45 & 0.43 & 0.40 & 0.39 & 0.38 & 0.37 & 0.37 & 0.37 & 0.37 & 0.37 \\
\hline 2.6 & 0.62 & 0.60 & 0.58 & 0.56 & 0.55 & 0.54 & 0.53 & 0.52 & 0.52 & 0.52 & 0.52 & 0.52 \\
\hline 2.2 & 0.34 & 0.33 & 0.32 & 0.30 & 0.30 & 0.29 & 0.28 & 0.28 & 0.28 & 0.27 & 0.27 & 0.27 \\
\hline 1.8 & 1.20 & 1.20 & 1.19 & 1.19 & 1.18 & 1.18 & 1.17 & 1.17 & 1.17 & 1.17 & 1.17 & 1.17 \\
\hline 1.35 & 0.19 & 0.18 & 0.18 & 0.18 & 0.18 & 0.18 & 0.18 & 0.17 & 0.17 & 0.17 & 0.17 & 0.17 \\
\hline 0.9 & 0.02 & 0.02 & 0.02 & 0.02 & 0.02 & 0.02 & 0.02 & 0.02 & 0.02 & 0.02 & 0.02 & 0.02 \\
\hline 0.4 & 0.07 & 0.07 & 0.07 & 0.07 & 0.07 & 0.07 & 0.07 & 0.07 & 0.07 & 0.07 & 0.07 & 0.07 \\
\hline
\end{tabular}

$\left.\left.\left.\left.\left.\left.\left.\left.\left.\left.\left.\left.\sum_{j}(2,3,3)\right|_{26.9}\right|_{2 . .9}\right|_{13.4}\right|_{22.3}\right|_{12.3}\right|_{10.7}\right|_{9.91}\right|_{9.27}\right|_{8.70}\right|_{8.40}\right|_{8.25}\right|_{8.24}$

The binding energy for a neutron in $49 \mathrm{Ti}$ is $8.146 \mathrm{MeV}$. 
TABLE VII

"DIRECT" EXCITATION OF LOW-LYING LEVELS IN NEUTRON CAPTURE IN Pb 206

\begin{tabular}{|c|c|c|c|c|c|c|c|c|}
\hline & 0.0 & .570 & .894 & 1.634 & 2.338 & 2.624 & 2.664 & 2.727 \\
\hline 8.85 & $.093^{a}$ & $.032^{a}$ & $.032^{\mathrm{a}}$ & $0^{b}$ & $0^{b}$ & $0^{b}$ & $0^{b}$ & $.742^{\mathrm{a}}$ \\
\hline 6.90 & $.250^{\mathrm{a}}$ & $.104^{a}$ & $.104^{2}$ & $.028^{\mathrm{b}}$ & $0^{b}$ & $0^{b}$ & $0^{b}$ & $.417^{\mathrm{a}}$ \\
\hline 5.37 & $.238^{a}$ & $.238^{b}$ & $.102^{\mathrm{b}}$ & $.046^{b}$ & $.034^{f}$ & $.034^{f}$ & $.034^{f}$ & $.171^{\mathrm{a}}$ \\
\hline 4.18 & $.272^{\mathrm{a}}$ & $.262^{c}$ & $.131^{\mathrm{c}}$ & $.054^{f}$ & $.033^{f}$ & $.033^{f}$ & $.033^{f}$ & $.087^{\mathrm{a}}$ \\
\hline 3.26 & $.243^{a}$ & $.280^{d}$ & $.134^{\mathrm{d}}$ & $.061^{f}$ & $.037^{\mathrm{f}}$ & $.037^{f}$ & $.037^{f}$ & $.074^{\mathrm{d}}$ \\
\hline 2.54 & $.392^{\mathrm{a}}$ & $.215^{\mathrm{d}}$ & $.103^{\mathrm{d}}$ & $.056^{f}$ & $.028^{f}$ & $.028^{f}$ & $.028^{f}$ & $.056^{\mathrm{d}}$ \\
\hline 1.78 & $.414^{\mathrm{a}}$ & $.198^{\mathrm{d}}$ & $.095^{\mathrm{d}}$ & $.069^{f}$ & $.026^{f}$ & $.026^{f}$ & $.026^{f}$ & $.043^{d}$ \\
\hline 1.08 & $.368^{f}$ & $.206^{f}$ & $.118^{\mathrm{f}}$ & $.074^{f}$ & $.029^{f}$ & $.029^{f}$ & $.029^{f}$ & $.044^{f}$ \\
\hline .604 & $.323^{f}$ & $.196^{f}$ & $.145^{f}$ & $.087^{f}$ & $.035^{\mathrm{f}}$ & $.035^{\mathrm{f}}$ & $.035^{f}$ & $.046^{f}$ \\
\hline .250 & $.242^{f}$ & $.182^{f}$ & $212^{f}$ & $.093^{f}$ & $.037^{f}$ & $.052^{f}$ & $.037^{f}$ & $.045^{f}$ \\
\hline .076 & $.161^{\mathrm{e}}$ & $.151^{\mathrm{e}}$ & $.303^{\mathrm{e}}$ & $.060^{f}$ & $.040^{f}$ & $.100^{\mathrm{e}}$ & $.044^{f}$ & $.044^{f}$ \\
\hline .028 & $.292^{e}$ & $.151^{\mathrm{e}}$ & $.154^{\mathrm{e}}$ & $.073^{\mathrm{f}}$ & $.041^{f}$ & $.107^{\mathrm{e}}$ & $.041^{\mathrm{f}}$ & $.041^{f}$ \\
\hline
\end{tabular}

Explanation of Superscripts
a. Read from Bergqvist's (Ref. 55) spectra
b. Estimated from Bergqvist's spectra
c. Estimated from Bergqvist giving $2 / 3$ of sum of .57 and .89 excitations
to $.57,1 / 3$ to .89
d. Extrapolated from Bergqvist's excitation functions
e. Calculated from data of Macklin (Ref. 34)
f. Interpolation as indicated in Fig. 2 
(2) Astronuclear

Waboratory

TABLE VIII

$\mathrm{Pb}^{206}$ CAPTURE - RADIANT ENERGY PER EVENT

\begin{tabular}{|l|l|l|l|l|l|l|l|l|l|l|l|l|}
\hline $\begin{array}{c}\text { Upundary } \\
\gamma \text {-Group }\end{array}$ & 1 & 2 & 3 & 4 & 5 & 6 & 7 & 8 & 9 & 10 & 11 & 12 \\
\hline $17.5 \mathrm{MeV}$ & 1.45 & 0 & 0 & 0 & 0 & 0 & 0 & 0 & 0 & 0 & 0 & 0 \\
\hline 15.5 & .951 & 3.41 & 0 & 0 & 0 & 0 & 0 & 0 & 0 & 0 & 0 & 0 \\
\hline 13.5 & 9.55 & 3.02 & 5.63 & 0 & 0 & 0 & 0 & 0 & 0 & 0 & 0 & 0 \\
\hline 11.5 & .034 & 4.56 & 1.96 & 6.99 & 2.43 & 0 & 0 & 0 & 0 & 0 & 0 & 0 \\
\hline 9.5 & .123 & .079 & 2.30 & 2.07 & 4.66 & 6.80 & 5.83 & 2.88 & 0 & 0 & 0 & 0 \\
\hline 7.5 & .057 & .041 & .033 & .024 & 1.09 & .006 & .003 & 1.49 & 2.37 & 0 & 0 & 0 \\
\hline 7.0 & .159 & .123 & .106 & .081 & .070 & .973 & .659 & 1.29 & 2.27 & 4.15 & 2.04 & 2.92 \\
\hline 6.0 & .232 & .192 & .177 & .144 & .133 & .118 & .653 & .738 & .714 & .532 & 2.13 & 1.31 \\
\hline 5.0 & .299 & .261 & .253 & .215 & .205 & .189 & .198 & .184 & .681 & .860 & 1.06 & 1.06 \\
\hline 4.0 & .314 & .284 & .285 & .249 & .244 & .229 & .245 & .245 & .238 & .249 & .237 & .243 \\
\hline 3.0 & .105 & .095 & .185 & .169 & .176 & .149 & .149 & .156 & .170 & .206 & .298 & .313 \\
\hline 2.6 & .076 & .068 & .069 & .062 & .061 & .058 & .063 & .063 & .061 & .064 & .063 & .066 \\
\hline 2.2 & .378 & .234 & .203 & .159 & .161 & .132 & .125 & .132 & .143 & .158 & .197 & .197 \\
\hline 1.8 & .041 & .038 & .100 & .094 & .101 & .083 & .083 & .088 & .097 & .102 & .106 & .109 \\
\hline 1.35 & 1.31 & .777 & .373 & .234 & .219 & .181 & .175 & .181 & .197 & .202 & .165 & .174 \\
\hline 0.9 & .514 & .448 & .436 & .427 & .442 & .352 & .337 & .369 & .400 & .461 & .517 & .391 \\
\hline 0.4 & 6.001 & $<.001$ & $<.001$ & $<.001$ & $<.001$ & $<.001$ & $<.001$ & $<.001$ & $<.001$ & $<.001$ & $<.001$ & $<.001$ \\
\hline
\end{tabular}


TABLE IX

TRANSITION PROBABILITY FROM CAPTURING STATE

To Level

ground state

$E_{x}=2.6 \mathrm{MeV}$

$3.2 \mathrm{MeV}$

$3.5 \mathrm{MeV}$
Calculated by CASCADE $\left(E_{n}=28 \mathrm{keV}\right)$

.318

.086

.058

.047
Used in Final Run

.461

.166

.043

.032

:

: 


\author{
APPENDIX I \\ INPUT INSTRUCTIONS FOR VERSION II OF THE CASCADE CODE
}

Card No. 1

Punch in Columns:

1-80 TITLE Alphanumeric description of this particular isotope. This information will be written on the gamma-ray spectra tape. It should contain information that is unique.

Card No. 2

1-5 N The number of excited levels to be input for this isotope. Do not count the ground state. The maximum number the code will handle is 50 .

6-10 NGP The number of points in the $\gamma(E)$ table. This table can have as few as 3 points or as many as 100.

11-15 IM The number of panels into which the continuum is divided in solving for $Q(E)$. This energy mesh, which is used in solving the differential equation, is computed by

$$
E_{k}=E_{m}+k \Delta E \quad k=0,1,2, \ldots . I M
$$

where $\Delta E=\frac{\epsilon-E_{m}}{I M}$

MI $\leq 980-3 N$ 
The number of panels into which the continuous part of the spectrum is divided. This energy mesh is computed by

$$
E_{\mathbf{r}}^{\mathrm{k}}=\mathrm{k \Delta E} \quad \mathrm{k}=0,1,2, \ldots . \mathrm{KK},
$$

where $\Delta E=\frac{\epsilon}{\mathrm{KK}}$

$\mathrm{KK} \leq 980-\left[\frac{3}{2} \mathrm{~N}(\mathrm{~N}+3)+2\right]$

For each $S(I, J)=0$ the maximum value of $\mathrm{KK}$ may increase by 3 .

21-25 IORC The type of calculation.

$$
\begin{aligned}
\text { IORC } & =0 \text { inelastic scattering } \\
& =1 \text { capture }
\end{aligned}
$$

26-30 INRO The option, for a capture calculation, to input or calculate the primary transitions $s_{i}^{0} ' s$.

INRO $=0 \quad s_{i}^{0}$ 's are calculated by: $S_{i}^{0}=f(\epsilon)\left(\epsilon-E_{i}\right)^{3}$

INRO $=1$ The $\alpha^{i}$ 's are input and the $s_{i}^{0}$ 's are calculated by: $s_{i}^{0}=\gamma(\epsilon) \alpha^{i}$

INRO should be zero when IORC $=0$.

31-35 IPLOT Plotting options

$$
\begin{aligned}
\text { IPLOT } & =0 \text { no plots } \\
& =1 \text { Plot } R(E) ; \text { the probability of excitation } / \mathrm{MeV} \\
& =2 \text { Plot } P\left(E_{\gamma}\right)-\text { photons } / \mathrm{MeV} / \mathrm{reaction}
\end{aligned}
$$


$=3$ Plot $P_{C}\left(E_{\gamma}\right)$ and $P_{D}\left(E_{\gamma}\right)$ e.g., separate curves for the continuum to continuum and the continuum to discrete.

$=4$ Plot all, 1.e., options 1, 2, and 3.

36-40 NEPS The number of $\epsilon$ 's we will have for this computer run. If IG $\neq 0$ (i.e., $\in$ 's are not input), NEPS may be left blank.

41-45 IOPTN Input the initial excitation probabilities, $r_{i}^{0} s$, or calculate them for an inelastic scattering calculation. This option applies only to cases where $\epsilon<E_{m^{*}}$ If all values of $\epsilon$ are such that $\epsilon>E_{m}$, IOPTN may be left blank. IOPTN $=1$ the $r_{1}^{0}$ 's are calculated by

$$
\begin{aligned}
& r_{i}^{0}=\left(\epsilon-E_{i}\right) / \sum_{j=1}^{k}\left(\epsilon-E_{j}\right) \\
& r_{0}^{0}=0
\end{aligned}
$$

IOPTN $=2$ the $r_{i}^{0}$ 's are calculated by $r_{i}^{0}=\sigma^{i}(\epsilon) / \sum_{j=1}^{k} \sigma^{j}(\epsilon)$ and the $\sigma^{i}(\epsilon)$ are input values. For $\epsilon>E_{m}$ the options are determined by the value of $E_{a}$, - see Card 3 . 
46-50 IG Indicator that tells the code how the $E$ 's are to be defined.

IG $=0$ Not a group calculation. Values of $\epsilon$ will be input into CASCADE. For each $\in$ the results will be a fine $\gamma$-ray cross-section spectrum. The code does not prepare a tape for CASING, 1.e., $\gamma$-ray production group cross sections cannot be obtained with this option.

IG $=1 \quad$ A group calculation where the neutron energy boundaries are input. The $\epsilon$ 's are calculated. Th1s option prepares a CASING tape.

$=-1 \mathrm{~A}$ calculation where neutron energy points are input and the $\epsilon$ 's are calculated. Prepares a CASING tape.

If IG $\neq 0$, one must request a blank tape on FORTRAN Logical Unit 10.

51-55 G If IG $=1$, this is the number of neutron groups. If IG $=-1$, this is the number of single neutron energies. If IG $=0, G$ may be left blank.

56-60 IROE Indicator that tells the code which nuclear level-density function to use.

$=1$ Expression based on the Fermi gas model. $\rho(E)=b e^{2 \sqrt{a E}}$ where, $a$ and $b$ are input quantities. 
$=2$ Expression for the Gilbert and Camerons density function; Formula A.

$$
\rho(E)=\left\{\begin{array}{l}
\frac{\alpha}{T} e^{\frac{E-E_{0}}{T}} \quad E \leq E_{X} \\
\frac{\beta}{\left(E-U_{0}\right)^{5 / 4}} e^{2 \sqrt{a\left(E-U_{0}\right)}} E>E_{X}
\end{array}\right.
$$

where $\alpha, E_{0}, E_{X}, T, \quad a$, and $U_{O}$ are input quantities.

$=3$ Expression for the Gilbert and Camerons density function; Formula B.

$$
\begin{aligned}
& \rho(E)=\left\{\begin{array}{lc}
\frac{\alpha}{T} e^{\frac{E-E_{O}}{T}} E \leq E_{X} \\
b\left(E-U_{0}\right)^{-\frac{3}{2} e^{2 \sqrt{a\left(E-U_{O}\right)}}} E>E_{X}
\end{array}\right. \\
& \text { where } \alpha, a, b, E_{0}, U_{0}, T \text {, and } E_{X} \text { are input } \\
& \text { quantities. }
\end{aligned}
$$

Card No. 3
a
b
Parameters that determine the nuclear level-density.
$b$ not needed if IROE $=2$. If $b$ is input as zero, and IROE $=3$ the program will calculate $b$ using the formula below:

$11-20$ 
$b=0.198 \alpha(a)^{-\frac{1}{2}}(A W)^{-\frac{1}{3}}$ where $\alpha, a$, and AW are input quantities.

$21-30$

$E_{m}$

Lower boundary of the continuum.

$31-40$

AW

The mass of the target nucleus in units of the neutron mass.

$41-50$

BE

The binding energy of the neutron in the residual nucleus. If the calculation is for inelastic scatter, $B E$ should be input as zero.

$51-60$

EA

$\mathrm{E}_{\mathrm{a}}$ For $\mathrm{E}>\mathrm{E}_{\mathrm{a}}$ option $\mathrm{S}-\mathrm{I}-\mathrm{I}$ is used.

For $E_{m}<E \leq E_{a}$ option S-I-2 is used.

These options are discussed in Part II.

Card Group No. 3a (Input this card only if IROE $\neq 1$ )

1-10 ALPHA $\propto$

11-20 BATA $\beta \quad$ (not needed for IROE $\neq 2$ )

21-30 EX EX Energy of tangency point when using the composite level density of Gilbert and Cameron.

$\begin{array}{ccc}31-40 & \text { EO } & E_{0} \\ 41-50 & \text { UO } & U_{0} \\ 51-60 & \text { T } & T\end{array}$




$\begin{array}{ll}\text { Card Group No. } 4 \mathrm{a} & \text { (Input this card group only if } I G=0 \text { ) } \\ \text { FORMAT(7E10.4) } & \text { EPSS Input NEPS } \in \text { 's. They should be in descending } \\ & \text { order, i.e., the highest value } \epsilon \text { should be } \\ & \text { input first. }\end{array}$

Card Group No. $4 \mathrm{~b}$ FORMAT(7E10.4)

Card Group No. $4 \mathrm{c}$ FORMAT(7E10.4)

Card Group 5

FORMAT(7E10.4)
(Input this card group only if IG = I)

EN Input $G+I$ neutron-group energy boundaries. They should be in descending order.

(Input this card group only if IG $=-1$ )

E Input G single neutron-energies. They should be in descending order.

EI Energies of the nuclear levels. The $E_{1}$ 's should be input in ascending order and the vector should contain $\mathrm{N}+1$ values; 0.0 should always be input as the first element in the vector.

Card Group 6

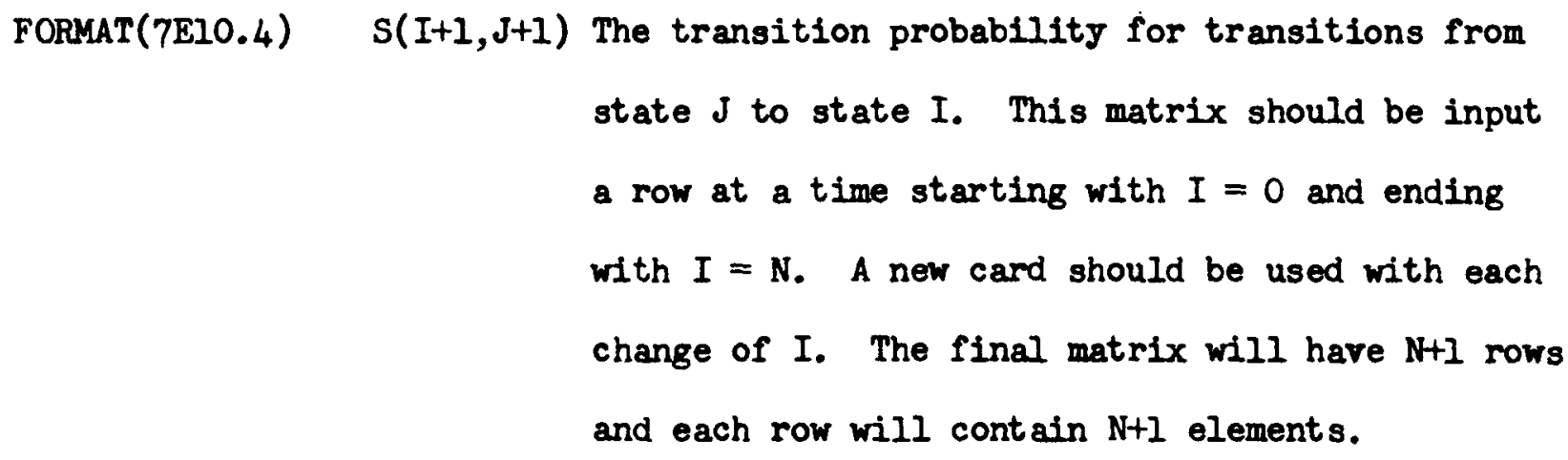
state $J$ to state I. This matrix should be input a row at a time starting with $I=0$ and ending with $I=N$. A new card should be used with each change of I. The final matrix will have $\mathrm{N}+\mathrm{l}$ rows and each row will contain $\mathrm{N}+1$ elements. 
Particular input rules below:

1. $\mathrm{I}+1>\mathrm{J}+1 \quad \mathrm{~S}(\mathrm{I}+\mathrm{I}, \mathrm{J}+\mathrm{I})=0.0$

2. $I+1=J+1 \quad S(I+1, J+I)=1.0$

3. $I+1<J+1 \quad S(I+I, J+I)=S^{j i}$

The lower triangular matrix elements will be equal to zero, and the diagonal elements will equal unity.

Card Group No. 7 FORMAT (7E10.4)

Input NGP energy values that are in correspondence with the $\gamma(E)$ vector. This energy vector should be input in ascending order. The last value in the EG array should be greater than the highest $\epsilon$ the program will use.

Card Group No. 8 FORMAT(7E10.4) GAMA Input NGP $\gamma(E)$ values.

Card Group No. 9 FORMAT(7E10.4)
(Input this card group only if INRO = I)

RO Input $(\mathrm{N}+\mathrm{I}) \alpha_{i}$ values. This vector is used to calculate the $s_{i}^{0}$ rector when doing a capture calculation, i.e.,

$$
s_{i}^{0}=\alpha_{i} \gamma(\epsilon)
$$

Insert in ascending order. 
Card Groups 10, 11, and 12 should onlJ be input when at least one of the $\in$ 's is greater than $E_{m}$. Also, if IPLOT is equal to zero, these card groups should not be input.

Card Group No. 10 FORMAT (6A10)

FOPMAT (6AIO)

FORMAT (6AlO)
(Input only if IPLOT is equal to 1 or 4)

TITIE Input any 48 characters. These 48 characters wlll be the title for this graph. This graph will be a graph of $R(E)$.

TITA Input any 54 characters. These characters will be the label on the abscissa of this graph.

TITO Same as TITA except these characters are for the ordinate.

Card Group No. 11 (Input only if IPIOT is equal to 2 or 4)

Input TITIE, TITA, and TITO as in card group 10 except this graph is a plot of $P\left(E_{\gamma}\right)$.

Card Group No. 12 (Input only if IPLOT is equal to 3 or 4)

Input TITLE, TITA, and TITO as in card group 10 except this graph is a plot of two curves $P_{C}\left(E_{\gamma}\right)$ and $P_{D}\left(E_{\gamma}\right)$.

The remaining card groups should only be input if IOPTN $=2$ and at least one $\epsilon$ is less than or equal to $E_{m}$. 
Card Group No. 13

Punch in Colums:

1-5 NSIG The number of points in the set of $\sigma^{i}(E)$ that is input below. $\quad 3 \leq$ NSIG $\leq 50$

Card Group No. 14

FORMAT(7E10.4) ESIG A set of energies in the range of $E_{1} \leq E \leq E_{m}$. These energies will be input in ascending order and there will be NSIG values for each ith level.

Card Group No. 15

$$
\text { FORMAT(TE10.4) SIG The corresponding } \sigma^{i}(E) \text { to the E's above. }
$$

Input the lowest $i^{\text {th }}$ level first and repeat card groups 13, $\mu_{4}$, and 15 for each level until the $\mathrm{N}^{\text {th }}$ level is input. There will be $\mathrm{N}+\mathrm{l}$ levels and each level will contain NSIG values. Be sure to start a new card for each card group.

If there are values of $\epsilon$ between $E_{m}$ and $E_{a}, N+2$ levels must be input. The last one being the cross section, $\sigma^{\Sigma}$, that is used to calculate $r_{i}^{0}$, i.e.

$$
r_{i}^{0}=\frac{\sigma^{i}(E)}{\sigma^{\Sigma}(\epsilon)}
$$

NSIG, ESIG, and SIG must be input for this cross section. These quantities are entered as they would be for the excitation cross section of one of the discrete levels. 
APPENDIX II

\section{INSTRUCTIONS FOR THE USE OF TRANSTT}

TRANSIT is a small $(\sim 25 \mathrm{~K})$ code that uses the single particle transition model of Moszkowski ${ }^{(36)}$ to estimate relative nuclear electromagnetic transition probabilities from a given initial state to several final states. The final states are selected by the user. The excitations, spins and parities of all participating states must be known. Each transition is assumed to go only by the lowest allowed multipolarity. The most elementary form of the transition probability was used, i.e., $P=C_{1} A^{C_{2}} E_{\gamma}^{C_{3}}$ where $A$ is the atomic weight of the nuclous, $E_{\gamma}$ is the energy difference between levels, and the constants $C_{1}, C_{2}$ and $\mathrm{C}_{3}$ depend on the multipolarity of the lowest allowed transition. The crudeness of this approach is acknowledged, but it is felt that some systematic method of guessing at decay probabilities of levels for which they weren't measured was preferable to omitting these levels altogether. At the user's option, these estimates may be modified by the Goldhaber-Sunyar empirical rotardation factors (42) which are derived from comparison of many transitions of each multipolarity with the single particle strength estimates. (e.g., experimental transition rates quoted by Goldhaber and Sunyar for about 40 E3-transitions rary between $\sim 0.05$ and $\sim .0005$ times the single particle estimate. Hence the E3 retardation factor is $\sim .005)$.

The crudeness of this entire approach is acknowledged but this program should give at least a rough idea of which transitions may be expected to be the strongest. 
Agreement with experiment for the decay of five states in $\mathrm{Pb}^{206}$ was fair for both the single particle and the retarded estimates.

\section{INPUT INSTRUCTIONS}

\section{Case I A Odd}

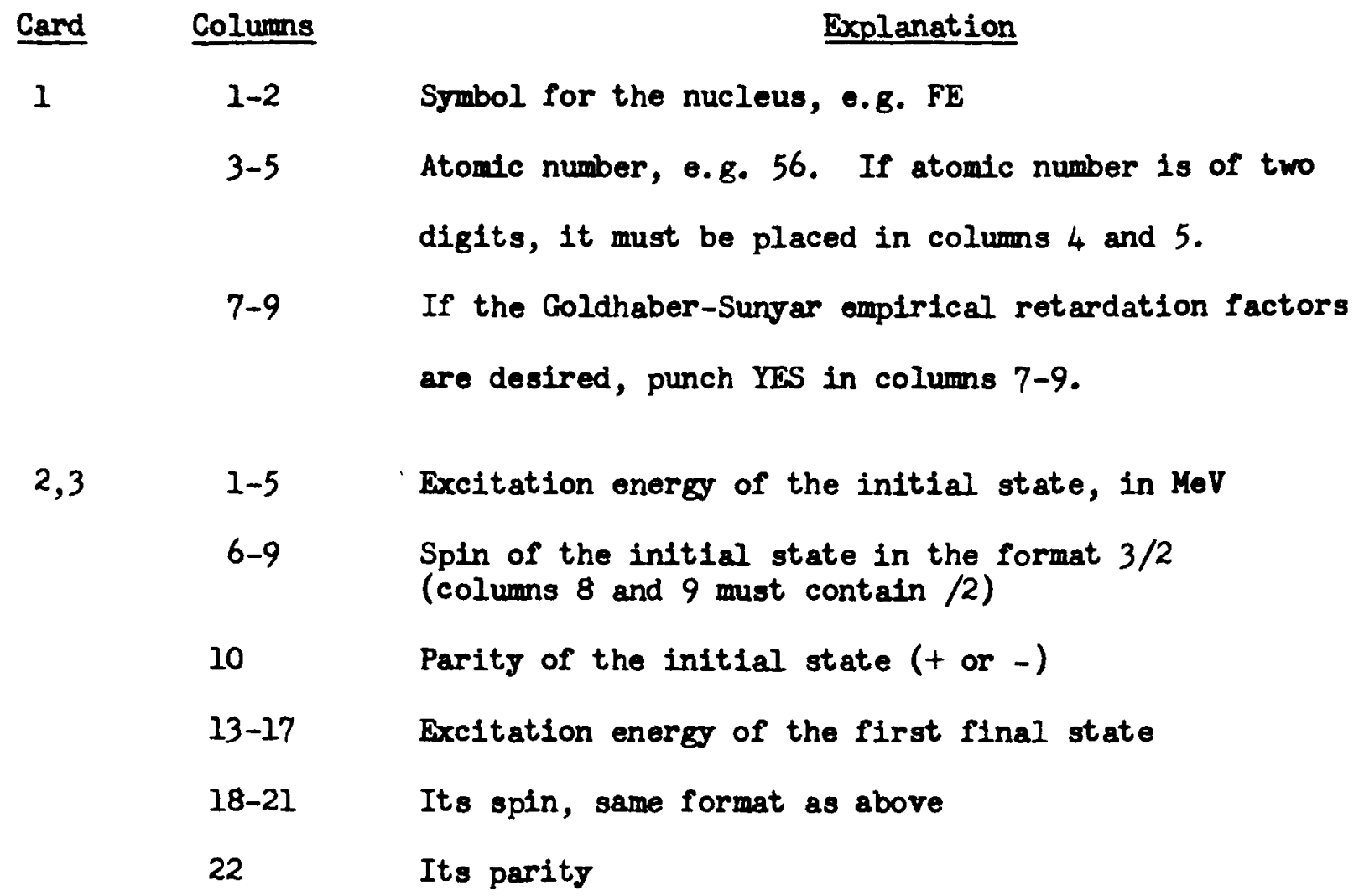

This format is repeated for each final state the user thinks is likely to be excited in the decay of the initial state, up to SEVEN FINAL STATES. The program will read $8 \times 12=96$ spaces independent of the number of final states, and as a card 3 must be included even though it will be blank if 5 or fewer final states are input.

$2 a, 3 a$

Same as 2,3 for another initial state. This pattern is repeated $(2 b, 3 b, \ldots$. .) until initial states are exhausted. 
W Astronuclear

Laboratory

Card $\quad$ Colwns $\quad$ Explanation

\section{Case II A Eron}

\begin{tabular}{|c|c|c|}
\hline Card & Colune & Bxplanation \\
\hline 1 & & Same as Case I \\
\hline \multirow[t]{6}{*}{2} & $1-5$ & Excitation energs of the initial state, in $\mathrm{MeV}$ \\
\hline & 7 & Spin of the initial state \\
\hline & 8 & Parity of the initial state \\
\hline & $11-15$ & Excitation energy of the first final state \\
\hline & 17 & Its spin \\
\hline & 18 & Its parity \\
\hline
\end{tabular}

This format is ropeated for each final state the user thinks is likely to be excited in the decay of the initial state, up to SEVEN FINAL STATES.

$2 \mathbf{a}$

Same as 2 for another initial state. This pattern is repeated $(2 b, 2 c, \ldots .$.$) until initial states are$ oxhausted.

3

A blank card

\section{GENERAL NOTES}

Jobs may be stacked. Just start again with card 1.

At the vory end of the entire run another blank card must be inserted for the program to exit properly. 
SAMPIE INPUT

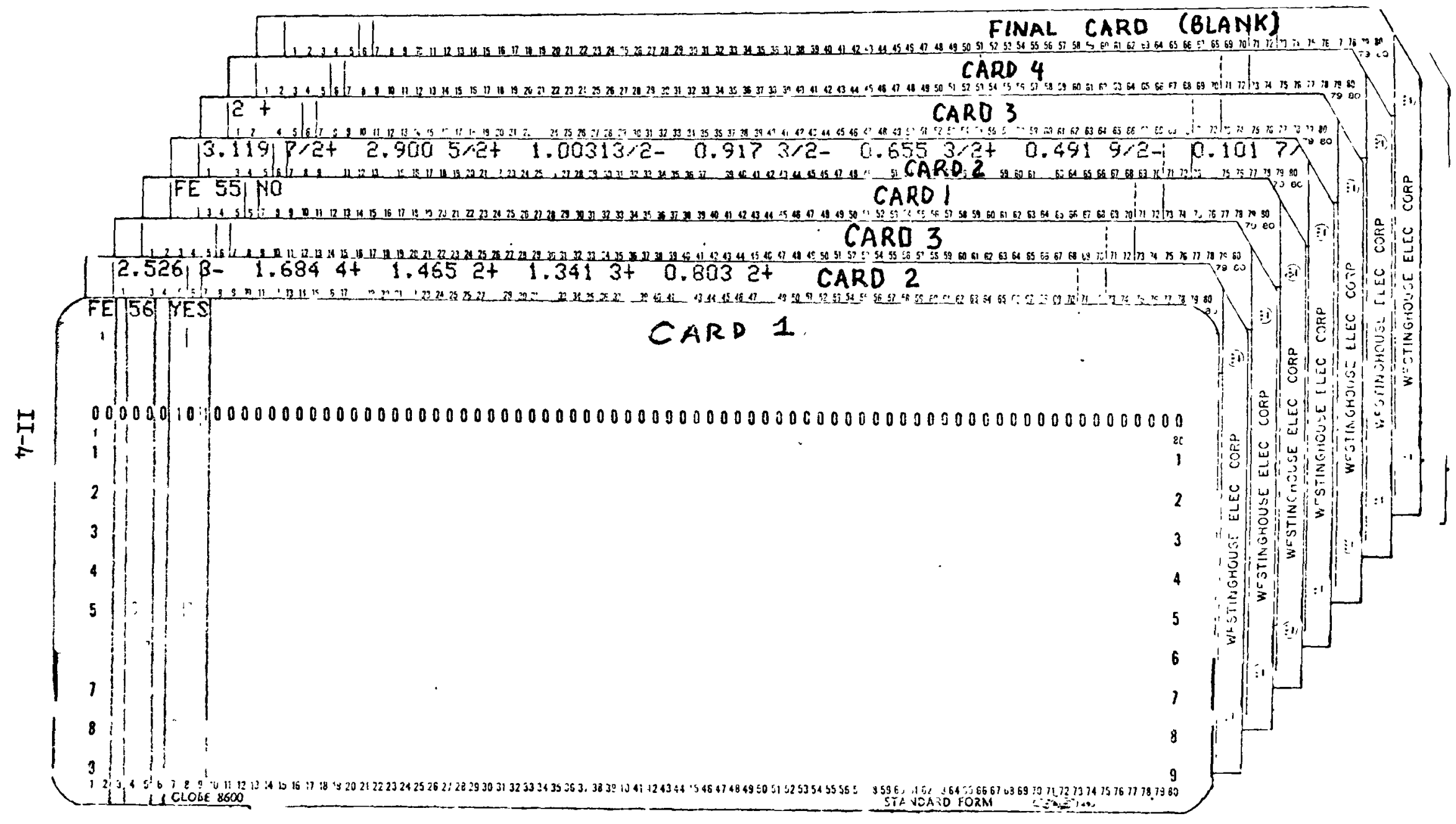




\subsection{REFERENCES}

1. G. Gibson and G. Collier, WANL-MME-2744, September (1970)

2. A. Gilbert and A. G. W. Cameron, Can. J. Phys. 43, 1446 (1965)

3. A. M. Lane and J. E. Ljun, Nucl. Phys. 17, 563, 586 (1960)

4. S. C. Mathur, W. E. Tucker, R. J. Benjamin and I. L. Morgan, Nucl. Phys. 73, 561 (1965)

5. J. H. Towle and W. B. Gilboy, Nucl. Phys. 39, 300 (1962)

6. Landolt-Bornstein Tables Vol. 1, K. H. Hellwege editor, Springer-Verlag (1961)

7. W. E. Kinney and F. G. Perey, ORNL-4516, October (1970)

8. S. K. Penny, ORNL-TM-2590, August (1969)

9. G. D. Joanou and C. A. Stevens, NASA CR-54260, GA-5884, November (1964)

10. Forsberg L., Aktiebolaget Atomenergi Stockholm (1963)

11. S. 0. Moore, BNL-927 (T-383) EANDC(US)-78, June (1965)

12. D. T. Goldman and C. R. Rubity, KAPI-2163, August (196I)

13. J. L. Perkin, Nucl. Phys. 60, 561 (1964)

14. John R. Stehn et al, BNL-325, 2nd Ed., Supp. 2, Vol. I (1964)

15. W. E. Ford III, CTC-42, O.R, N. L., September (1970)

16. W. E. Kinney and F. G. Perey, ORNL-4249, August (1968)

17. P. F. Hinrichsen, M. H. Shapiro, and D. M. Van Patter, Nucl. Phys. Al01, 81 (1967)

18. D. M. Drako, J. C. Hopkins, and C. S. Young, Nucl. Sci. and Eng. 40, 294 (1970)

19. J. R. Macdonald and M. A. Grace, Nucl. Phys. A92, 593 (1967)

20. R. J. Benjamin, P. S. Buchanan, and J. L. Morgan, Nucl. Phys, 29, 241 (1966)

21. A. Aspinwall, G. Brown, and S. E. Warren, Nucl. Phys. 46, 33 (1963) 
22. M. H. Shapiro, P. F. Hinrichsen and R. Middleton

23. H. K. Vonach and J. R. Huizenja, Phys. Rev. 138, B1372 (1965)

24. P. C. Gugelot, Phys. Rev. 81, 51 (1951)

25. Neutron Physics Division (Annual Progress Report Ending May 31, 1969) ORNL-4433, p. 44, September (1969)

26. "Table of Isotopes", C. M. Lederer, J. M. Hollander and I. Perlman, Wiley and Sons, Inc., 1967

27. E. D. Earle et al, Physics Letters 32B, No. 6, 471 (1970)

28. J. Towle and W. Gilboy, Nucl. Phys. 44, No. 2, 256 (1963)

29. S. O. Moore and E. H. Auerbach, BNL-818 (T-317), 1963

30. B. R. S. Buckingham et al, "Neutron Cross-Sections of Selected Elements and Isotopes for Use in Neutronics Calculations in the Energy Range $0.025 \mathrm{eV}-15 \mathrm{MeV} "$, AWRE, No. $0-28 / 60,1960$

31. R. A. Moyer et al, Phys. Rev. C2, 1898 (1970)

32. D. O. Nellis, Texas Nuclear Div, , ORD-2791-27, p. 103 (1968) and private communication

33. G. Vallois et al, Phys. Letters 22, 659 (1966)

34. R. L. Macklin et al, Phys. Rev. 136, B695 (1964)

35. D. E. Alburger and A. W. Sunyar, Phys. Rev. 29, 695 (1955)

36. K. Siegbahn, ed., Alpha-, Beta-, and Gamma Ray Spectroscopy, 1965, p. 863

37. L. Cranberg et al, Physical Review 159, 969 (1967)

38. S. O. Moore, Brookhaven National Laboratory, BNI-50151 (T-520) (1968)

39. F. D. Bechetti Jr. and G. W. Greenlees, Phys. Rev. 1182, 1190 (1969)

40. P. S. Buchanan, A Compilation of Cross Sections and Angular Distributions of Gamma Rays Produced by Neutron Bombardment of Various Nucleil, Texas Nuclear Corporation, OR0-2791-28 (1969)

41. E. R. Shunk, R. T. Wagner, and A. Kemmendinger, Buzl. Am. Phys. Soc. I, 334 (1962) 
42. K. Siegbahn, ed., Alpha-, Beta- and Gamma Ray Spectroscopy, 1965, p. 931

43. J. Solf et al, Nuclear Physics Al39, 523 (1969)

44. G. Vallois et al, Physics Letters 24B, 512 (1967)

45. E. R. Flynn et al, Physical Review Letters 19, 798 (1967)

46. D. E. Alburger and M. Pryce, Physical Review 95, 4882 (1954)

47. C. J. Herrlander, Arkiv for Fysik, 20, 71 (1961)

48. M. D. Goldberg et al, Brookhaven National Laboratory, BNL-325, Second Edition, Supplement 2, Volume 2C, 1966, p. 82-206-3

49. M. S. Moore, ed., Reports to the AEC Nuclear Cross Sections Advisory Conmittee, WASH-1124, 1968, p. 186

50. P. Fettweis and M. Saidane, Nucl. Phys. Al39, 113 (1969)

51. Nuclear Data Section A, 3, Nos. 4-6, 469 (1967)

52. S. Fody, WANL-TME-1946, August (1969)

53. N. C. Rasmussen et al, MITNE-85, Scientific Report No. 2, January (1969)

54. J. A. Biggerstaff et al, Physical Review 154, 1136 (1967)

55. I. Bergqvist et al, Nuclear Physics Al53, 553 (1970)

56. M. B. Lewis, Nuclear Data Sheets 5, 247 (1971) 


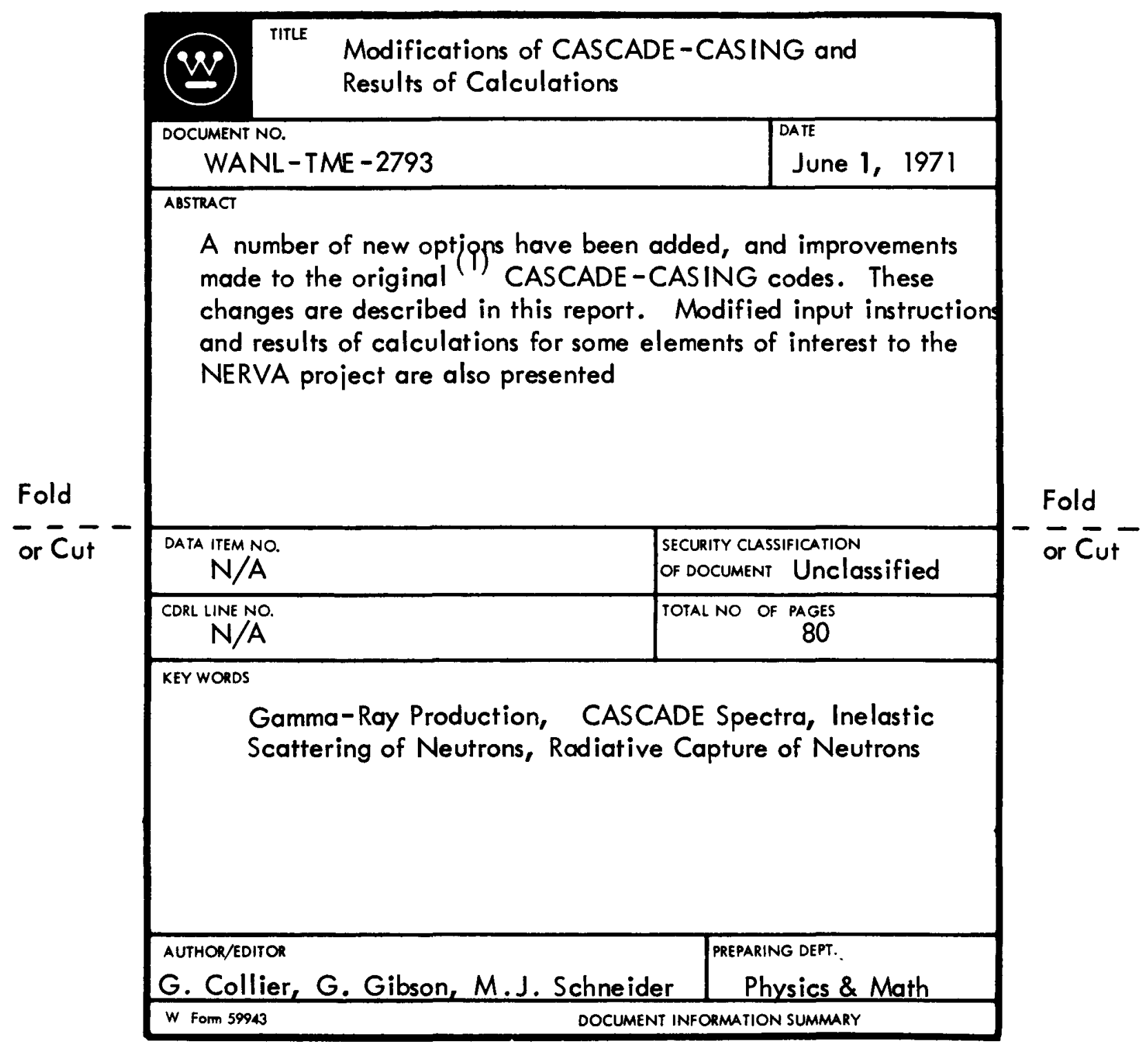

